A N N A L E S Annales de Bretagne et des Pays de l'Ouest

\title{
Les Templiers en Bretagne au Moyen Âge : mythes et réalités
}

\section{Philippe Josserand}

\section{(2) OpenEdition}

\section{Journals}

Édition électronique

URL : http://journals.openedition.org/abpo/2488

DOI : $10.4000 /$ abpo. 2488

ISBN : 978-2-7535-2236-7

ISSN : 2108-6443

Éditeur

Presses universitaires de Rennes

Édition imprimée

Date de publication : 31 décembre 2012

Pagination : 7-33

ISBN : 978-2-7535-2234-3

ISSN : 0399-0826

\section{Référence électronique}

Philippe Josserand, «Les Templiers en Bretagne au Moyen Âge : mythes et réalités », Annales de Bretagne et des Pays de l'Ouest [En ligne], 119-4 | 2012, mis en ligne le 31 décembre 2014, consulté le 30 avril 2019. URL : http://journals.openedition.org/abpo/2488 ; DOI : 10.4000/abpo.2488 


\title{
Les Templiers en Bretagne au Moyen Âge : mythes et réalités
}

\author{
Philippe JOSSERAND \\ Maître de conférences en histoire du Moyen Âge, université de Nantes, CRHIA
}

Les Templiers n'ont jamais complètement disparu de Bretagne. Après la suppression de leur ordre décidée par le concile de Vienne en $1312^{1}$, différentes traditions se sont attachées à eux, glosées parfois et ressassées jusqu'à aujourd'hui, entre lesquelles il n'est pas toujours facile pour l'historien de se mouvoir. Connus en particulier sous le nom de "moines rouges", les frères du Temple sont demeurés vivants dans la mémoire collective bien au-delà du Moyen Âge, fantômes inquiétants, condamnés à errer pour expier d'affreux et abominables crimes. Théodore Hersart de La Villemarqué, qui édita en 1839 le Barzaz-Breiz - Chants populaires de la Bretagne, témoigna que, de son temps, le petit peuple croyait voir encore, la nuit, les " moines rouges ": "Ils sont vêtus de manteaux blancs et portent une grande croix écarlate sur la poitrine; ils montent des squelettes de chevaux enveloppés dans des draps mortuaires. Ils poursuivaient, dit-on, jadis les voyageurs, s'attaquant de préférence aux petits garçons et aux jeunes filles, qu'ils enlevaient et conduisaient Dieu sait où, car ils ne les ramenaient point ${ }^{2}$. " Aux préoccupations folkloristes du XIX ${ }^{\mathrm{e}}$ siècle, la période actuelle en a ajouté d'autres, notamment touristiques, qui n'ont pas réellement contribué, dans ce que l'on peut lire et consulter sur la Bretagne, à débarrasser les Templiers des oripeaux de légende dont ils sont de longue date affublés.

Pourtant, une historiographie du Temple existe en Bretagne, hélas souvent méconnue, et depuis le milieu du XIX ${ }^{\mathrm{e}}$ siècle des recherches rigoureuses ont été menées à bien sur le passé de l'ordre. Jusqu'alors, dans les histoires du duché, de Bertrand d'Argentré à Pierre-Antoine Daru, la présence des frères n'avait nullement intéressé ${ }^{3}$. La situation changea, en

1. Demurger, Alain, Les Templiers. Une chevalerie chrétienne au Moyen Âge, Paris, 2005, p. $466-467$.

2. Hersart de La Villemarqué, Théodore, Barzaz Breiz. Chants populaires de la Bretagne, Paris, 4e éd., 1846, p. 312.

3. ARGENTRÉ, Betrand d', Histoire de Bretagne, des rois, ducs, comtes et princes d'icelle, Rennes, 2 vol., 1582, et DARU, Pierre-Antoine, Histoire de Bretagne, Paris, 3 vol., 1826. 
lien avec le renouveau de la science historique, autour de 1850, grâce à des érudits comme Paul de La Bigne-Villeneuve et Anatole de Barthélemy, qui, les premiers, s'attachèrent à recenser les possessions templières en Bretagne $^{4}$. Ce fut cependant, à la charnière des XIX et XX ${ }^{\mathrm{e}}$ siècles, Amédée Guillotin de Corson, chanoine de la cathédrale de Rennes et président de la Société archéologique d'Ille-et-Vilaine de 1886 à 1888, qui s'illustra plus que tout autre en ce domaine, publiant en 1902 un livre appelé Les Templiers et les Hospitaliers de Saint-Jean de Jérusalem en Bretagne, dans lequel il reprenait et développait les textes d'une série d'articles parus au cours des vingt années précédentes ${ }^{5}$. Plusieurs fois réédité, l'ouvrage, en dépit de ses manques et de son intérêt concentré en priorité sur l'Ancien Régime, reste aujourd'hui la base de toute étude sérieuse portant sur les Templiers en Bretagne, et force est de reconnaître que le $\mathrm{xx}^{\mathrm{e}}$ siècle lui a très peu apporté, le renouvellement de la recherche s'étant uniquement esquissé au cours des dernières années, dans le cadre universitaire, notamment grâce à JeanChristophe Cassard et à François Colin ${ }^{6}$.

Pas plus que du temps d'Amédée Guillotin de Corson, il n'est facile aujourd'hui d'écrire l'histoire du Temple en Bretagne. Comme l'a souligné le chanoine dès les premières lignes de son avant-propos, "l'ordre a disparu depuis si longtemps que ses archives sont devenues extrêmement rares; ses monuments sont tombés en ruines; ses traditions n'offrent plus guère qu'incohérence et incertitude ${ }^{7}$ ". Est-ce à dire que l'historien doit renoncer et abandonner le public, souvent intéressé par ce qui touche aux Templiers, entre les mains d'affabulateurs et d'adeptes de l'ésotérisme? Le " sottisier templier ", pour reprendre la belle expression d'Alain Demurger ${ }^{8}$, n'est pas une fatalité, et il revient aux historiens, creusant le sillon labouré par Amédée Guillotin de Corson, de démontrer que le passé des frères en Bretagne peut être étudié de façon scientifique, comme l'a été celui des Cisterciens ou des Mendiants, Franciscains

4. La Bigne-Villeneuve, Paul de, "Sur les possessions des Templiers en Bretagne ", Bulletin archéologique de l'Association bretonne, 4 (1852), p. 188-202, et BARTHÉLEMY, Anatole de, "Chartes de Conan IV, duc de Bretagne, relatives aux biens de l'ordre du Temple et de l'ordre de Saint-Jean de Jérusalem ", Bibliothèque de l'École des Chartes, 33 (1872), p. 445-456.

5. Guillotin de Corson, Amédée, Les Templiers et les Hospitaliers de Saint-Jean de Jérusalem en Bretagne, Nantes, 1902, rééd. Marseille, 1987, puis sous le titre Les Templiers en Bretagne et les Hospitaliers de Saint-Jean de Jérusalem, Fouesnant, 2006. C'est à cette dernière édition, accessible à défaut d'être optimale, qu'il sera fait référence par la suite.

6. CASSARD, Jean-Christophe, L'Orient des Bretons au Moyen Âge, Morlaix, 2007, et CouIN, François, "Templiers et Hospitaliers en Bretagne du XII ${ }^{\mathrm{e}}$ au début du XIV ${ }^{\mathrm{e}}$ siècle. Histoire, historiographie et mythe ", Mémoire de première année de master, université de Nantes, 2007.

7. Guillotin de Corson, Amédée, Les Templiers en Bretagne et les Hospitaliers de SaintJean de Jérusalem, p. 23.

8. DEMURGER, Alain, "Introduction au dossier : L'étude des ordres religieux-militaires en France : la fin de la marginalité? ", Cahiers de Recherches Médiévales. A Journal of Medieval Studies, 15 (2008), p. 169. 
et Dominicains ${ }^{9}$. Pour appréhender le Temple dans le duché, il existe des documents médiévaux, moins nombreux certes que pour d'autres ordres, mais dont il convient absolument de partir, avant d'aller vers tout ce qui peut faire sens. Indispensable, la recherche en archives et en bibliothèques ne saurait seule suffire, et elle doit s'ouvrir aux acquis de l'archéologie, de l'analyse du bâti, de l'étude de la toponymie ou de la tradition pour avoir une chance d'offrir une juste vision de l'implantation des Templiers en Bretagne. Telle est en tout cas la logique qui m'a guidé dans ces pages, au parti pris scientifique desquelles j'espère que le lecteur sera sensible, la saveur de l'histoire du Temple valant bien celle de ses prétendus mystères ${ }^{10}$.

\section{Près de deux siècles d'histoire}

\section{L'établissement des Templiers en Bretagne}

L'ordre du Temple est né en 1120 à l'initiative d'un petit seigneur champenois, Hugues de Payns, qui allait en devenir le premier maître ${ }^{11}$. C'est à Jérusalem qu'il fut fondé, l'institution se donnant pour but, par les armes, de protéger les pèlerins et de défendre les États latins issus de la première croisade. Pour prétendre durer, la jeune communauté devait s'assurer le soutien matériel, institutionnel et spirituel de l'Occident et, en particulier, de l'Église, et c'est à cette fin qu'Hugues de Payns et quelques-uns de ses frères, en 1127, quittèrent la Terre sainte, entreprenant un périple de deux ans qui leur permit d'obtenir la reconnaissance de l'ordre au concile de Troyes en janvier 112912. De Provence en Écosse et d'Anjou en Champagne, les Templiers purent alors se constituer d'importants appuis, et la Bretagne ne semble en rien avoir fait exception au mouvement. Très tôt, les frères s'y sont en effet établis. La première donation consentie au Temple dans le duché appert d'un acte intéressant Coudrie, en Bas-Poitou, siège d'une future commanderie (fig. 1). Un seigneur breton figure au nombre des bienfaiteurs de l'ordre, Garsire, sire de Retz et de Machecoul, qui, outre un cheval et des armes, céda avec l'accord de sa femme et son fils du foin pris sur ses prés et diverses rentes assises à Pornic et à Bouin ${ }^{13}$. La charte n'est pas datée, mais un document postérieur indique que ces dons ainsi

9. MARTIN, Hervé, Les ordres mendiants en Bretagne (vers 1230-vers 1530), Paris, 1975, et DuFIEF, André, Les Cisterciens en Bretagne aux XII et XIII siècles, Rennes, 1997.

10. La matrice de ce travail, dépourvue d'appareil critique, est parue sous la forme d'un petit livre aux éditions Gisserot : JossERAND, Philippe, Les Templiers en Bretagne, Paris, 2011. Je tiens à remercier vivement l'éditeur, et en particulier Christophe Renault, auteur des photographies de l'ouvrage, pour m'avoir autorisé à en reproduire un certain nombre ici.

11. Leroy, Thierry, Hugues de Payns, chevalier champenois, fondateur de l'ordre des Templiers, Troyes, 2001, p. 65-67.

12. Demurger, Alain, Les Templiers. Une chevalerie chrétienne au Moyen Âge, p. 51-66.

13. LA BoutetiÈre, Louis de, "Cartulaire de Coudrie ", Archives historiques du Poitou, 2 (1873), p. 153-154, doc. 1. 
que d'autres en Poitou avaient été remis " dans la main de maître Hugues de Payns ${ }^{14}$ ", et il est donc probable qu'ils remontent au printemps 1128 , époque où le dignitaire est attesté dans l'Anjou voisin ${ }^{15}$.

\section{Figure 1 - La chapelle de la commanderie de Coudrie (cliché Chr. Renault)}

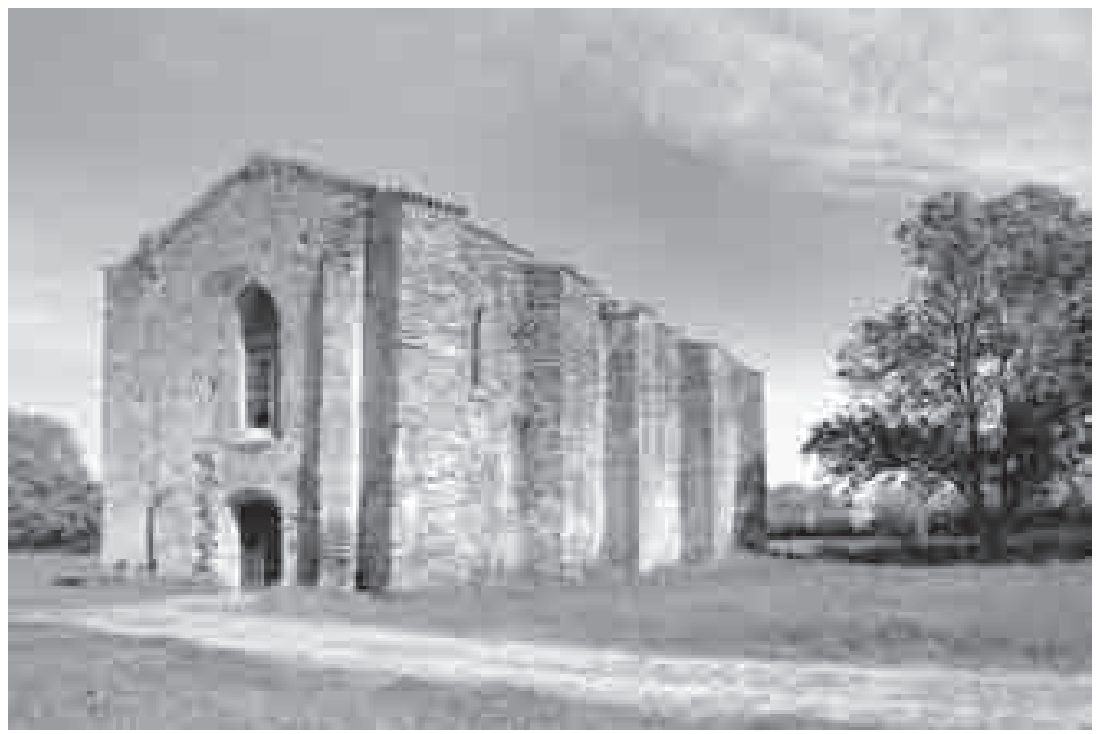

La même année, sans doute, une autre charte, aux effets plus considérables, allait sceller l'implantation définitive des Templiers en Bretagne ${ }^{16}$. Elle fut octroyée, à Nantes, par le duc Conan III, sa mère, Ermengarde, sa femme, Mathilde, et sa fille Berthe. Ensemble, ils donnèrent aux frères l'île de la Hanne, en amont de la ville, sur la Loire; ils leur cédèrent aussi une métairie " avec ses maisons et ses animaux " dans la forêt de Rennes, Mathilde ajoutant les biens qu'elle possédait à proximité, et le duc, en vertu de son pouvoir princier, leur confirma par avance toutes les terres qu'ils acquerraient dans son domaine ${ }^{17}$. L'acte, hélas, n'est pas daté, mais une autre charte, issue en 1141, permet de le situer. Cette année-là, Conan III consentit de nouveaux dons aux Templiers et, dans son privilège, il commença par rappeler la cession passée de l'île de la Hanne (fig. 2), indiquant

14. Ibid., p. 155-158, doc. 2 : "in manu magistri Ugonis de Paganis ".

15. Demurger, Alain, Les Templiers. Une chevalerie chrétienne au Moyen Âge, p. 53.

16. Guillotin DE CoRson, Amédée, Les Templiers en Bretagne et les Hospitaliers de SaintJean de Jérusalem, p. 28-29.

17. Barthélemy, Anatole de, et Geslin de Bourgogne, Jules, Anciens évêchés de Bretagne. Histoire et monuments, Saint-Brieuc, t. vI, 1879, p. 121-122. 
que les autres faveurs étaient intervenues ensuite (postea) ${ }^{18}$. La première charte ducale, dénuée de date, est donc antérieure à 1141. Amédée Guillotin de Corson, le premier, proposa de la faire remonter à l'époque du séjour d'Hugues de Payns en Occident ${ }^{19}$ et, peut-on préciser, eu égard à ce que l'on sait aujourd'hui du périple, au printemps 1128. Le maître du Temple a-t-il jamais rencontré le duc de Bretagne, sollicitant directement faveur et protection pour son ordre? En l'état des sources, on ne saurait l'assurer, mais l'hypothèse sans conteste tient du vraisemblable.

\section{Figure 2 - La charte de Conan III, de 1141, donnant aux Templiers l'âle de la Hanne (cliché J.-M. Roger)}

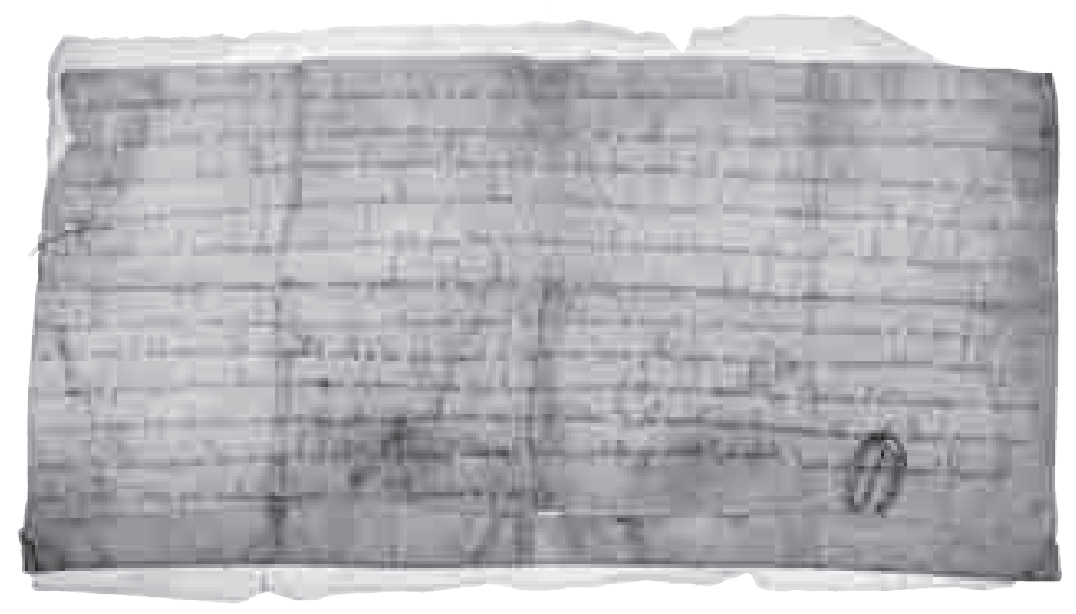

En 1141, cinq ans après la disparition d'Hugues de Payns ${ }^{20}$, Conan III décida de compléter les largesses qu'il avait naguère accordées au Temple. Désormais conduit par Robert de Craon, l'ordre était représenté en Occident par Guillaume Faucon, le maître cismarin, chargé à ce titre de la collecte des aumônes consenties à l'institution ${ }^{21}$ : c'est ce dernier dignitaire, accompagné de deux frères, Alfred et Henri, comme lui étrangers à la Bretagne, qui reçut la nouvelle donation ducale. Celle-ci consistait en une rente annuelle de cent sous à prélever la première semaine de Carême

18. Morice, Hyacinthe, Mémoires pour servir de preuves à l'histoire ecclésiastique et civile de Bretagne, Paris, t. I, 1742, col. 583, et BARTHÉLEMY, Anatole de, et GeSLIN DE Bourgogne, Jules, Anciens évêchés de Bretagne, t. VI, p. 122-123.

19. Guillotin de Corson, Amédée, Les Templiers en Bretagne et les Hospitaliers de SaintJean de Jérusalem, p. 28.

20. LEROY, Thierry, Hugues de Payns, p. 91.

21. On voit ce dignitaire intervenir pour la première fois en Champagne, en 1133 (AlBON, Guigues d', Cartulaire général de l'ordre du Temple (1119?-1150). Recueil des chartes et des bulles relatives à l'ordre du Temple, Paris, 1913, p. 43). 
sur les droits de marché du faubourg de Nantes et surtout en un terrain, au Pré d'Anian, destiné à être construit (plateam ad domos sive edificium), où allait s'élever la future commanderie urbaine, en rive droite de l'Erdre, dans un espace intégré à l'enceinte sous Pierre de Dreux ${ }^{22}$. Outre ces dons, Conan III prit les biens du Temple sous sa protection dans l'ensemble du duché et il leur accorda de pouvoir jouir d'exemptions de taxes reconnues par ses principaux barons. Ainsi l'implantation des frères s'est-elle ancrée dans la durée. Amorcée dès la fin de la décennie 1120, elle a largement bénéficié des largesses du duc et de son entourage, mais, au-delà de Conan III et du sire de Retz et de Machecoul, ce sont de vastes secteurs de la société bretonne, souvent bien mal documentés, qui se sont montrés favorables au Temple et en ont soutenu le développement.

\section{Un accueil favorable au sein du duché}

En 1148, la mort de Conan III, qui avait abondamment appuyé les Templiers, précipita la Bretagne dans une longue période de troubles. Le duc ayant déshérité son fils Hoël et choisi pour lui succéder le jeune Conan IV, né de sa fille Berthe, des forces nombreuses s'élevèrent contre le pouvoir princier : les principaux barons reprirent leurs revendications d'autonomie et, à l'extérieur comme à l'intérieur du duché, Plantagenêt et Capétiens s'affrontèrent jusqu'à l'avènement d'une nouvelle dynastie, celle des Dreux, en $1213^{23}$. Dans un contexte aussi incertain, on pourrait penser que le Temple n'a guère pu compter sur la libéralité des ducs. Il n'en fut rien. Une charte de 1217, en vertu de laquelle Pierre de Dreux et son épouse, Alix, ratifiaient les dons de leurs prédécesseurs, manifeste que pratiquement tous ceux qui prétendirent au pouvoir ducal au XII ${ }^{\mathrm{e}}$ siècle ont favorisé l'ordre : il est question de Conan III, bien sûr, mais aussi d'Hoël, son fils déshérité, qui occupa le comté de Nantes, d'Alain le Noir, père de Conan IV, de ce dernier ainsi que de Geoffroy II et de Constance ${ }^{24}$. Aucune de ces concessions n'est précisée, et seule de la dernière, on possède encore le texte ${ }^{25}$. Toutefois, la tradition ducale d'appuyer les Templiers était ancrée, et Pierre de Dreux ne fit que lui rendre toute sa force, concédant à l'ordre plus de privilèges que celui-ci n'en avait jamais reçu, dans un esprit qu'allaient perpétuer ses fils et petit-fils, Jean I ${ }^{\text {er }}$ et Jean II ${ }^{26}$, intéressés comme lui par la croisade en Terre sainte ${ }^{27}$.

Favorisés par le pouvoir ducal, les Templiers, jusqu'à la fin de leur présence en Bretagne, bénéficièrent aussi du soutien d'amples secteurs de la

22. Tonnerre, Noël-Yves, "Le haut Moyen Âge (V $-\mathrm{xIII}{ }^{\mathrm{e}}$ siècles) ", dans Histoire de Nantes, éd. par Paul Bois, Toulouse, 1977, p. 72.

23. CASSARD, Jean-Christophe, La Bretagne des premiers siècles. Le Haut Moyen Âge, Paris, 1994, p. 79-84.

24. Morice, Hyacinthe, Mémoires pour servir de preuves, t. I, col. 836.

25. Arch. dép. de la Vienne, 3H1 764.

26. Colin, François, "Templiers et Hospitaliers en Bretagne ", p. 34-36.

27. Jones, Michael, "Les Bretons et la croisade ", Mémoires de la Société Historique et Archéologique de Bretagne, 61 (1994), p. 373-375. 
société locale. Toutes les couches semblent avoir participé de l'élan de générosité envers les frères. Parmi les bienfaiteurs de l'ordre, on relève des individus d'humble condition, paysans ou simples bourgeois, tels Pierre Lévin, qui vers 1200 fit don d'une terre à la Laurière, en Port-Saint-Père ${ }^{28}$, ou Daniel Le Barrilier, qui avec les siens céda en 1233 une rente de vingt sous sur le Pont-Neuf d'Erdre à Nantes ${ }^{29}$; des clercs figurent également au nombre des promoteurs du Temple, à l'image d'Arbert Moraut, en $1215^{30}$, ou d'Olivier Gaudin, au début du XIV ${ }^{\mathrm{e}}$ siècle encore ${ }^{31}$. Néanmoins, comme en d'autres régions, la plupart des donateurs étaient issus de la noblesse. Les grands barons du duché, que Conan III déjà avait voulu associer à ses largesses, et, plus encore, la moyenne noblesse et la simple chevalerie constituèrent des appuis essentiels pour le Temple. Ce dernier secteur, en particulier, était en étroite consonance spirituelle avec l'ordre, qui y a largement recruté, recevant par exemple en 1197 Hamelin du Pinel, seigneur du Pinel, en Argentrédu-Plessis, au diocèse de Rennes, qui, devenu veuf, se fit Templier, cédant à ses nouveaux coreligionnaires, avec l'assentiment de son fils, une dîme qu'il ordonna de partager avec les Cisterciens de Clermont ${ }^{32}$.

Participant comme Cîteaux des courants de réforme qui, à partir de la fin du $\mathrm{XI}^{\mathrm{e}}$ siècle, se sont développés au sein de l'Église, le Temple était regardé avec beaucoup de faveur ${ }^{33}$. La Bretagne ne faisait nullement exception. Donner à l'ordre était une ouvre pie que les bienfaiteurs espéraient utile au salut de leur âme et de celles de leurs proches, certains, parfois, spécifiant davantage leurs attentes, comme Raoul de Machecoul, qui, en 1212, déclara agir en mémoire de son frère, décédé depuis peu ${ }^{34}$, et par crainte de la fugacité du monde ${ }^{35}$. Par-delà ces éléments de piété génériques, qui pouvaient bénéficier à d'autres institutions religieuses, le Temple fut favo-

28. Barthélemy, Anatole de, et Geslin de Bourgogne, Jules, Anciens évêchés de Bretagne, t. VI, p. 182.

29. Ibidem, p. 171.

30. La BouteTiÈRe, Louis de, "Cartulaire de Coudrie ", p. 187-188, doc. 35.

31. Barthélemy, Anatole de, et Geslin de Bourgogne, Jules, Anciens évêchés de Bretagne, t. VI, p. 222.

32. Arch. dép. de la Vienne, registre 497 (terrier de la commanderie de La Guerche de 1776), p. 1608-1609 : "Noverit universitas vestra quod Hamelinus de Pinello, miles, ad religionem Templariorum transiens, assensu et concessione filii sui, Hugonis, dedit et concessit integre sine aliqua retentione dilectis fratribus monachis de Claro Monte et Templariis, pro salute animae suae et antecessorum suorum, quidquid habebat in decima de Verauceio, quam in parrochia de Argenteio possidebat".

33. CARRAZ, Damien, L'ordre du Temple dans la basse vallée du Rhône (1124-1312). Ordres militaires, croisades et sociétés méridionales, Lyon, 2005, p. 133-187, et ID., "Les ordres militaires et hospitaliers : une "nouvelle religion" ", dans Structures et dynamiques religieuses dans les sociétés de l'Occident latin (1179-1449), éd. par Jean-Michel Matz et MarieMadeleine de Cevins, Rennes, 2010, p. 179-180 et 184-186.

34. LA BouteTIÈRE, Louis de, "Cartulaire de Coudrie ", p. 181, doc. 26 : " pro salute anime mee et redemptione anime fratris mei Bernardi, omniumque predecessorum meorum ".

35. Ibidem, p. 181-183, doc. 27 : "labentem mundum cito pretereuntem aspiciens et in meis bonis operibus nunquam confidens, sed de malis bonis actibus timens, pro adhipiscenda venia peccatorum meorum et pro remedio animarum patris et matris mee et tocius generis mei". 
risé par l'intérêt ancien qui existait en Bretagne pour la Terre sainte ${ }^{36}$. Alain Fergent, le père de Conan III, avait participé à la première croisade, et par la suite nombre de seigneurs bretons continuèrent à prêter main-forte à l'Orient latin ${ }^{37}$. Deux d'entre eux, d'outre-mer, consentirent un don aux Templiers : André de Vitré, engagé en 1184 dans l'opération lancée contre Saladin pour débloquer le château de Kérak, à l'est de la mer Morte ${ }^{38}$, et Philippe de Vigneux, combattant au siège de Damiette, en $1219^{39}$. Au début du XIV ${ }^{\mathrm{e}}$ siècle encore, Jérusalem éveillait certaine résonance en Bretagne, et le Temple en tirait parti, auquel, dans son testament de 1302, le duc Jean II remit un legs, à partager avec l'Hôpital, pour qu'il « soit mis et converti au proffilt e en l'aide de la sainte Terre ${ }^{40}$ ".

\section{Un développement tronqué par le procès}

Au tournant des $\mathrm{XIII}^{\mathrm{e}}$ et XIV ${ }^{\mathrm{e}}$ siècles, en dépit de l'éviction des Latins de Terre sainte, la popularité des Templiers restait réelle. Le développement des frères en Bretagne, s'il s'était assez largement ralenti, n'avait pas été remis en cause, et de crise, là comme ailleurs, plus souvent qu'on ne l'écrit, il n'en exista pas avant l'ouverture du procès. Ce dernier, dans sa genèse, est extérieur à la Bretagne, puisqu'il est né de la volonté de Philippe IV le Bel et de ses légistes ${ }^{41}$. Le 14 septembre 1307, le souverain capétien ordonna à tous les baillis et sénéchaux de son royaume de préparer en secret l'arrestation des Templiers. Fixée au 13 octobre, celle-ci n'a pas épargné le duché gouverné alors par Arthur II. Le coup de filet, terrible, n'a pas laissé de trace dans les archives bretonnes, et seule la tradition populaire - comme Amédée Guillotin de Corson l'a souligné pour Carentoir ${ }^{42}$ - a pu en conserver un lointain écho, mais son impact est hors de doute. Dans le duché de Bretagne, les Templiers furent bel et bien arrêtés, comme ils l'ont été dans toute la France, et leurs biens furent, là aussi, placés sous séquestre $^{43}$. Le 3 mars 1308, Philippe IV commissionna deux chevaliers afin de recevoir les possessions locales de l'ordre et de les affermer ${ }^{44}$. Une tradi-

36. CASSARD, Jean-Christophe, L'Orient des Bretons au Moyen Âge, p. 116-133.

37. Jones, Michael, "Les Bretons et la croisade ", p. 367-370.

38. Morice, Hyacinthe, Mémoires pour servir de preuves, t. I, col. 691.

39. Barthélemy, Anatole de, et Geslin de Bourgogne, Jules, Anciens évêchés de Bretagne, t. VI, p. 159-160.

40. Morice, Hyacinthe, Mémoires pour servir de preuves, t. I, col. 1185-1191, en particulier col. 1189 .

41. THÉRY, Julien, "Procès des Templiers ", dans Prier et combattre. Dictionnaire européen des ordres militaires au Moyen Âge, éd. par Nicole Bériou et Philippe Josserand, Paris, 2009, p. 743-751, et ID., "Une hérésie d'État. Philippe le Bel, le procès des "perfides Templiers" et la pontificalisation de la royauté française ", Médiévales, 60 (2011), p. 157186.

42. Gulllotin de Corson, Amédée, Les Templiers en Bretagne et les Hospitaliers de SaintJean de Jérusalem, p. 119.

43. Colin, François, "Templiers et Hospitaliers en Bretagne ", p. 37-38.

44. MoRICE, Hyacinthe, Mémoires pour servir de preuves, t. I, col. 1216-1217 : " ad levandum, colligendum, exigendum et recipiendum omnia bona mobilia et immobilia quae fuerunt 
tion du XV $v^{\mathrm{e}}$ siècle rapporte que lorsque les hommes du roi vinrent à Nantes, le 10 août, ils en furent chassés ${ }^{45}$, mais, quand bien même cela aurait été, le Temple n'a pas échappé en Bretagne aux aléas du procès.

Pressé par le pouvoir royal qui s'employait à accréditer toutes sortes d'accusations contre les Templiers, le pape Clément V, par la bulle Faciens misericordiam, du 13 août 1308, se résolut à une solution judiciaire. Ainsi s'ouvrit une double procédure, qui devait pour les frères être jugée dans le cadre des diocèses et pour l'ordre à l'échelle de la chrétienté ${ }^{46}$. Des enquêtes bretonnes, il ne reste rien, et l'on est fondé à se demander si chacun des neuf évêchés du duché a réellement obéi à l'injonction du Saint-Siège. Seules trois dépositions de Templiers servant en Bretagne au moment de l'arrestation ont été conservées dans les interrogatoires de la commission pontificale siégeant à Paris au printemps 1310 : chacun des frères assura qu'il avait été entendu dans un premier temps à Poitiers, précisant bien qu'il avait alors été absous et réconcilié ${ }^{47}$. Pour ce qui est des crimes dont ils étaient accusés à l'égal de leurs coreligionnaires, Gérard d'Auguiniac, le commandeur de Nantes, Renaud Larchier et Pierre de La Nouée se défendirent avec force, le premier liant même les attaques contre l'ordre à la suspicion des " méchantes gens ${ }^{48}$ ". Dès lors, la déposition d'un Templier anglais prétendant que, dans la maison de Nantes, du blé était distribué aux porcs plutôt qu'aux pauvres fait figure de racontar ${ }^{49}$. En Bretagne, comme ailleurs, les frères étaient innocents, et ceux d'entre eux qui avaient été absous et réconciliés, une fois leur ordre dissous, le 3 avril 1312, purent achever leur existence dans les lieux où ils avaient vécu, ordinairement dévolus aux Hospitaliers ${ }^{50}$.

Considérant que les biens du Temple étaient destinés à aider la Terre sainte, Clément V, par la bulle Ad providam, du 2 mai 1312, décida de les réunir à ceux de l'Hôpital de Saint-Jean de Jérusalem, qui partageait le combat de son illustre devancier au contact duquel, à partir du milieu du XII ${ }^{\mathrm{e}}$ siècle, il s'était progressivement militarisé ${ }^{51}$. En Bretagne, comme dans le reste

et erant Templariorum patriae Britanniae [...] et ad concedendum domos ipsas, fructus, redditus et proventus ad firmam ".

45. Ibid., col. 1217 : "E est assavoir que les dous commissaires nommez en cest instrument, quand ils furent venus à Nantes pour cuider mettre à execution leur dite commission, furent chacez e boutez hors de Nantes vilainement par les bourgeois e autre gens notables demourans en icelle, disans que au roy n'apartenoit point avoir les biens d'iceux Templiers, ainçois apartenoient e estoient confisquez au duc de Bretaigne, leur prince e seigneur, e non à autre ".

46. Demurger, Alain, Les Templiers. Une chevalerie chrétienne au Moyen Âge, p. 448.

47. Michelet, Jules, Le procès des Templiers, Paris, t. II, 1987, p. 82-85, 105-107 et 188-189.

48. Ibid., t. II, p. 83 : "Clandestine recipiabantur, nullis presentibus nisi fratribus ordinis, ex quo credit quod per malos suspicio contra ordinem haberetur".

49. Ibid., t. I, p. 199 : "Dixit se vidisse in domo dicti ordinis, civitatis Nannatensis, quod Thomas, claviger dicte domus, frater dicti ordinis, dabat interdum porcis bonum bladum et pauperibus panem de fulfure".

50. Colin, François, "Templiers et Hospitaliers en Bretagne ", p. 41.

51. Demurger, Alain, Les Templiers. Une chevalerie chrétienne au Moyen Âge, p. 467-473. 
du royaume de France, les Hospitaliers, jusqu'alors moins puissants que les Templiers ${ }^{52}$, furent ainsi légalement désignés pour recevoir ce que ces derniers avaient possédé53. Le 28 mars 1313, le roi Philippe IV s'adressa au bailli de Tours, Jean de Vaucelles, qui envoya en Bretagne deux sergents pour opérer la dévolution. De ce transfert de biens, l'on garde un procèsverbal exceptionnel, publié il y a peu par Jean-Marc Roger, intéressant les établissements du Créac'h, de la Caillibotière et de la Nouée, aux diocèses de Saint-Brieuc et de Saint-Malo, où il eut lieu entre le 22 et le 24 mai $^{54}$. Aucun autre acte de ce type n'a, pour l'heure, été mis au jour, mais il ne fait pas de doute que la dévolution a dû s'opérer rapidement dans tout le duché : les rétentions forcées, évoquées parfois dans le diocèse de Nantes, furent au total plutôt rares ${ }^{55}$, et dès la fin juin 1313 une vente à Pont-d'Arm, en Assérac, fit mention d'une terre située " ès fès à l'Ospital, qui fut jadis au Temple $^{56}$ ». L'histoire des Templiers en Bretagne avait pris fin, celle de leurs possessions, plus importantes que l'on le pense parfois, se poursuivait, désormais au pouvoir de l'Hôpital.

\section{Une forte implantation régionale}

\section{La difficulté d'une pesée globale}

De l'importance de la présence templière en Bretagne, l'on a beaucoup débattu. Certains, notamment dans des publications marquées du sceau de l'ésotérisme, n'hésitent pas à la tenir pour considérable; d'autres, tel Louis-Christian Gautier, ont pu la juger modeste, au motif que les actes conservés, sous forme d'originaux ou de copies, sont en nombre limité et en tout cas bien moindre que pour d'autres espaces français, y compris

52. Guillotin de Corson, Amédée, Les Templiers en Bretagne et les Hospitaliers de SaintJean de Jérusalem, p. 48, et Colin, François, et Josserand, Philippe, "Bretagne ", dans Prier et combattre. Dictionnaire européen des ordres militaires au Moyen Âge, éd. par Nicole Bériou et Philippe Josserand, Paris, 2009, p. 175-176.

53. Une vision d'ensemble, demandant à être reprise, a été procurée jadis par DuvAL, Michel, "La dévolution des biens du Temple aux Hospitaliers en Bretagne ", dans Actes du $97^{e}$ Congrès national des Sociétés Savantes (Nantes, 1972). Assistance et assistés jusqu'au XVte siècle, Paris, 1979, p. 287-298.

54. Arch. nat. de France, M 1, n 14, publ. par Roger, Jean-Marc, " La prise de possession par l'Hôpital des maisons du Temple en Poitou et en Bretagne (mai 1313) ", Revue historique du Centre-Ouest, 7.2 (2008), p. 240-243, doc. 2. De cet acte, dont il existe une copie du milieu du XVIII ${ }^{\mathrm{e}}$ siècle (Arch. nat. de France, MM 3, $\mathrm{f}^{\circ}$ 91-97 v ${ }^{\circ}$ ), une transcription a également été procurée, à partir de cette dernière, par CoLIN, François, "Templiers et Hospitaliers en Bretagne ", p. 200-206.

55. L'analyse de CoLIN, François, "Templiers et Hospitaliers en Bretagne ", p. 44, amène à relativiser le point de vue de DuvaL, Michel, "La dévolution des biens du Temple aux Hospitaliers en Bretagne ", dans Actes du $97^{e}$ Congrès national des sociétés savantes. Philologie et histoire, Nantes, 1972, p. 287-298.

56. Arch. dép. de la Vienne, 3H1 781 : «Une pièce de terre arable avec ses appartenances, que ladite Nobile avoit e avoir poet, comme elle disoit, ès champ que l'on apele le clos Arabie, entre le cortil Pierres le Barber, d'une part, e la fontayne au sinlz pères, d'autre, ès fes à l'Ospital, qui fut jadis au Temple. " 
septentrionaux ${ }^{57}$. Prendre la mesure de l'implantation bretonne du Temple est difficile. Pourtant, l'historien qui s'intéresse à l'ordre localement ne peut s'en dispenser. Pour tenter une pesée globale, le document essentiel, publié pour la première fois par Anatole de Barthélemy, est une charte datée de 1182 et attribuée au duc Conan IV, énumérant sans aucunement les détailler une centaine de lieux où le Temple possédait des biens (fig. 3). L'acte, pour le Temple, est exceptionnel, mais, dans la forme comme sur le fond, il se rapproche d'un privilège que le même prince, en 1160, aurait conféré aux Hospitaliers, leur confirmant une soixantaine de lieux où ils détenaient des intérêts ${ }^{58}$. Indispensables à l'analyse des ordres militaires, dont ils offrent une idée de l'étendue des domaines en Bretagne, les deux documents ne sont connus qu'à l'état de copie, tardive, celle qui se rapporte au Temple, conservée dans un document de $1451^{59}$, posant plus de difficultés encore que celle dont l'Hôpital, depuis 1312, pouvait se prévaloir ${ }^{60}$.

Datée de 1182, la charte confirmative des biens du Temple en Bretagne est, sans conteste possible, apocryphe. Son découvreur, Anatole de Barthélemy, l'avait déjà souligné ${ }^{61}$. Certes, le seul frère dont il est fait état, Guillaume Ferron, paraît avoir exercé des responsabilités dans la région à l'époque, témoin, par exemple, d'une donation de Conan IV au MontSaint-Michel en $1170^{62}$, mais de graves problèmes chronologiques affectent l'acte et obligent à mettre en cause son authenticité, l'évêque de Léon, Hamon, et le duc lui-même étant morts depuis plus de dix ans au moment de sa concession supposée. En termes de critique diplomatique, le privilège octroyé au Temple n'est pas non plus exempt de soupçon. On ne connaît pas en Bretagne, excepté pour les ordres militaires, de chartes confirmatives des biens d'une institution contenant leur énumération; surtout, on peine à imaginer que le duc, en 1182, ait pu garantir aux frères des biens situés en dehors de son domaine, les grands fiefs, comme le Rohan ou le Penthièvre, étant alors assez autonomes. L'acte prétendant recenser l'ensemble des biens du Temple en Bretagne est donc un faux, de fabrication postérieure, mais, pour reprendre l'heureuse expression de François Colin, il s'agit d'un faux auquel, eu égard au caractère exceptionnel des informations qui en émanent, "l'historien doit faire confiance ", pour peu qu'une analyse soigneuse en soit effectuée ${ }^{63}$.

57. GAUTIER, Louis-Christian, Histoire des Templiers en Bretagne, Fouesnant, 2008, p. 51-52.

58. La première édition en a été faite par MoRICE, Hyacinthe, Mémoires pour servir de preuves, t. I, col. 638, sous le titre erroné de " charte du duc Conan IV pour les Templiers " que, quelque cent ans plus tard, La Bigne-Villeneuve, Paul de, "Sur les possessions des Templiers en Bretagne ", p. 188-202, a permis de corriger.

59. Arch. dép. de la Vienne, 3H1 764.

60. Arch. nat. de France, J241/A, n 26.

61. BARTHÉLEMY, Anatole de, "Chartes de Conan IV », p. 455.

62. Morice, Hyacinthe, Mémoires pour servir de preuves, t. I, col. 662-663.

63. Colin, François, "Quand l'historien doit faire confiance à des faux : les chartes confirmatives de Conan IV, duc de Bretagne, aux Templiers et aux Hospitaliers ", Annales de Bretagne et des pays de l'Ouest, 115.3 (2008), p. 33-55. 

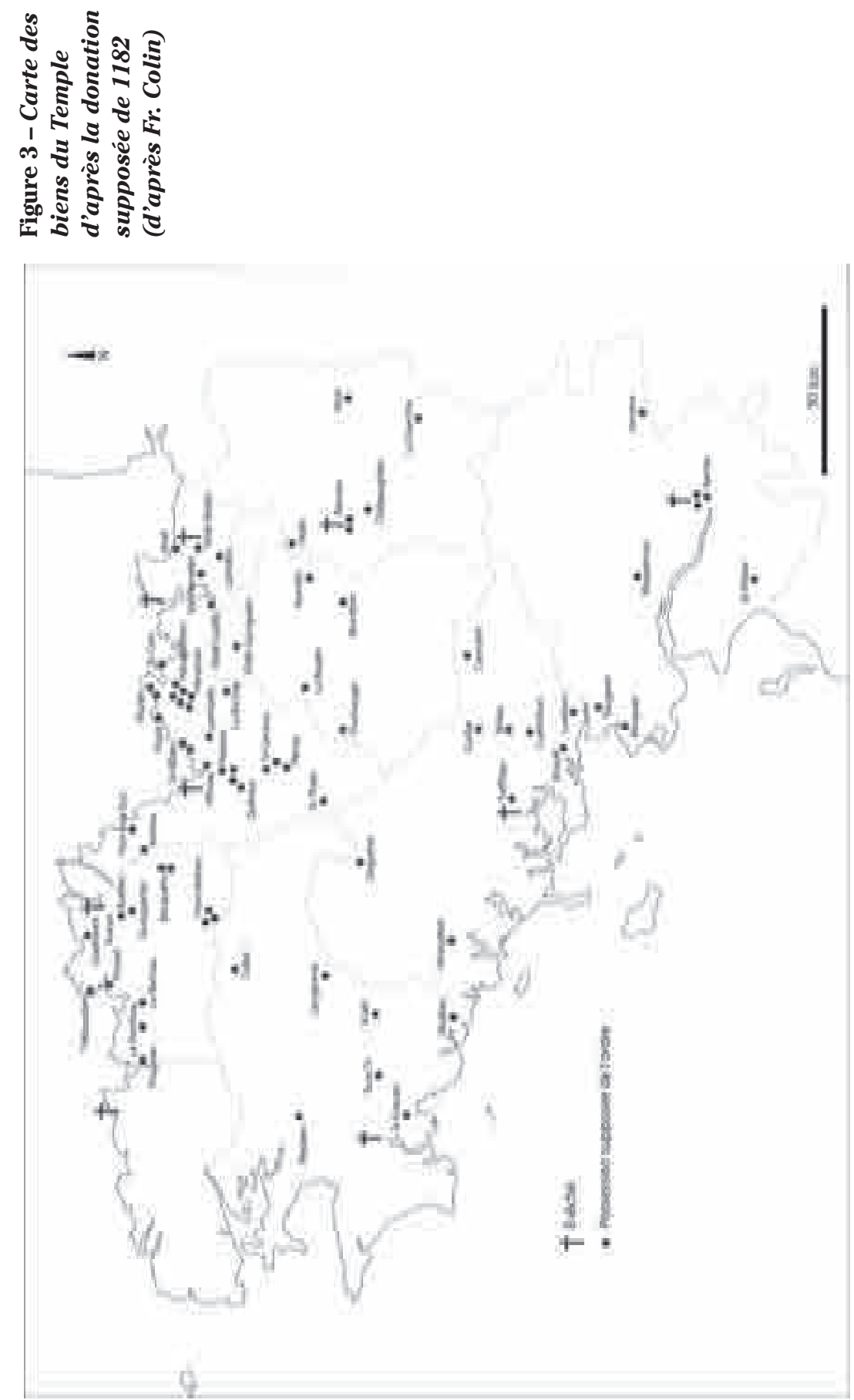
Fausses, les deux chartes en faveur du Temple et de l'Hôpital attribuées à Conan IV n'en avaient pas moins comme finalité d'être crues. Elles devaient donc être vraisemblables, et c'est en cela qu'elles intéressent l'historien, auquel elles offrent des éléments précieux. Le tableau qu'elles fournissent des possessions des ordres militaires en Bretagne est sans équivalent ${ }^{64}$. Une centaine de noms de lieux apparaissent dans l'acte consenti au Temple, moins soigneusement classés que dans le document de l'Hôpital qui, plus structuré, suit, dans l'énumération, le cadre diocésain. Anatole de Barthélemy a attribué jadis les deux faux à une même main et, parce que la charte de l'Hôpital, d'après la plus ancienne copie conservée, a été vidimée par l'évêque de Dol en 1277, il a suggéré que leur rédaction aurait été opérée vers $1270^{65}$. L'idée a été couramment acceptée, même si rien ne permet d'établir que le vidimus de 1277, invoqué en 1312, était authentique. C'est, animé d'un tel doute, que François Colin a récemment proposé de situer plutôt les deux forgeries autour de 1312, celle qui est censée bénéficier aux Templiers tenant de la " mauvaise $\operatorname{copie}^{66}$ ". L'hypothèse est tout à fait séduisante, et ce sont donc les Hospitaliers qui, pour asseoir leur position dans le contexte de la dévolution des biens du Temple, auraient fabriqué les deux faux, lesquels rendraient compte du patrimoine des ordres, non pas en 1270 - voire auparavant - mais au début du XIV ${ }^{\mathrm{e}}$ siècle.

\section{Des établissements entre ville et campagne}

Représenter au moyen du texte ou de la carte l'implantation du Temple en Bretagne reste aujourd'hui encore un défi. Le manque d'archives est un obstacle redoutable, accru par le fait que celles qui sont conservées offrent une vision déséquilibrée du temporel de l'ordre, privilégiant les diocèses de Nantes et de Rennes. Pour pallier ce problème, on a cherché, de longue date, à utiliser les actes d'Ancien Régime intéressant l'Hôpital, héritier du Temple, et toutes sortes de traditions populaires, empruntant au folklore ou à la toponymie. Amédée Guillotin de Corson, qui se méfiait de ces dernières, a beaucoup exagéré la continuité entre les biens de l'Hôpital aux XVII ${ }^{\mathrm{e}}$ et XVIII ${ }^{\mathrm{e}}$ siècles et ceux du Temple un demi-millénaire plus tôt, mais force est de constater que depuis lors la connaissance n'a pas avancé ${ }^{67}$. La tradition, comme l'a fait Michel Lascaux, a souvent été reprise sans regard critique ${ }^{68}$, et, récemment encore, Louis-Christian Gautier écrivait que les possessions du Temple en Bretagne affectaient « la

64. Guillotin de CoRson, Amédée, Les Templiers en Bretagne et les Hospitaliers de SaintJean de Jérusalem, p. 31.

65. BARThÉlemy, Anatole de, "Chartes de Conan IV ", p. 455-456.

66. Colın, François, "Quand l'historien doit faire confiance à des faux ", p. 44-46.

67. À un siècle de distance, le caractère essentiel du travail du chanoine a été souligné par JonEs, Michael, "Les Bretons et la croisade ", p. 378, et CASSARD, Jean-Christophe, L'Orient des Bretons au Moyen Âge, p. 148.

68. Lascaux, Michel, Les Templiers en Bretagne, Rennes, 1979. 
forme d'un fer à cheval dirigé vers l'est ", se demandant si cela constituait un symbole ${ }^{69} \ldots$ Pour l'historien, qui doit rejeter de telles élucubrations, la réalité est plus prosaïque : présents de manière ancienne et probablement plus forte dans le diocèse de Nantes, les Templiers se sont établis dans l'ensemble des évêchés bretons, excepté le Léon - le faux attribué à Conan IV mentionnant, par exemple, pour Saint-Brieuc douze paroisses à peine documentées par ailleurs -, sans que l'on puisse en déduire une quelconque chronologie d'implantation ${ }^{70}$.

Les donations octroyées au Temple en Bretagne, d'après ce que révèlent les actes, se sont échelonnées jusqu'à la veille du procès. Au milieu du XIII ${ }^{\mathrm{e}}$ siècle, toutefois, il est possible de tenir le temporel de l'ordre pour achevé dans ses grandes lignes ${ }^{71}$. En dépit du caractère imprévisible des aumônes, il ne s'est pas constitué au hasard. Des pressions ont pu être exercées sur des donateurs, tels Arbert Clérebaut, contraint d'abandonner une terre à la Roncinière, dans le Pays de Retz, vers $1166^{72}$, des échanges et des achats négociés avec d'autres, de façon à ce que le Temple puisse bâtir son domaine au mieux de ses intérêts ${ }^{73}$. Recherchant des profits capables d'être aisément mobilisés, pour aider au secours de la Terre sainte, les frères ont écarté le faire-valoir direct et se sont très tôt intégrés au système seigneurial, alors dominant. Leur gestion, mal connue, est exceptionnellement éclairée en 1257 par le rentier de la commanderie de Clisson : aux confins du duché avec l'Anjou et le Poitou, le Temple, largement investi dans la céréaliculture et l'élevage, semble s'être fait une spécialité de la vigne et de certaines activités de transformation, fortement rémunératrices, comme les fours et les moulins ${ }^{74}$. En d'autres lieux, il n'est pas impossible que le même souci de rentabilité ait prévalu, les frères développant les foires, comme aux Biais ${ }^{75}$, créant des fronts de défrichement, dans l'intérieur du Trégor, à partir de Pont-Melvez ${ }^{76}$, et suscitant, peut-être, des habitats nouveaux, à Moustérien, en Saint-Tugdual, ou dans plusieurs localités appelées Vildé

69. GAUTIER, Louis-Christian, Histoire des Templiers en Bretagne, p. 58.

70. Colin, François, " Templiers et Hospitaliers en Bretagne », p. 59-65.

71. Colin, François, et Josserand, Philippe, "Bretagne », p. 175.

72. LA Boutetiěre, Louis de, "Cartulaire de Coudrie ", p. 161, doc. 6.

73. Ibidem, p. 172-174 et 209-210, doc. 19 et 61, et BARTHÉLEMY, Anatole de, et GESLIN DE Bourgogne, Jules, Anciens évêchés de Bretagne, t. VI, p. 176.

74. Arch. dép. de la Vienne, 3H 729 bis (registre de vingt-huit folios), publ. par LE SAUCE, Xavier, «La commanderie de Clisson au Moyen Âge. Étude d'un établissement templier et hospitalier du duché de Bretagne (XII $-\mathrm{XV}^{\mathrm{e}}$ siècle) ", Mémoire de première année de master, Université de Nantes, 2006, t. II, p. 5-29.

75. LA BoutetièRe, Louis de, "Cartulaire de Coudrie ", p. 172-174, en particulier p. 173, doc. 19 : "Dominus Archodius Templariis concessit nuundinas suas domus dau Bierz, quas per violentiam illis abstulerat, ut in ipso loco restitueret ac in die dominica ante Ascensionem Domini faceret residere; atque per octonos dies post et antea alteras nundinas in tota terra sua non coadunaret, per quas prefate nundine pejores essent, quia fratres Templi de dono Constancie, comitisse Britannie, has nundinas habuisse referebant".

76. LAURENT, Jeanne, Un monde rural en Bretagne au $X v^{e}$ siècle : la quévaise, Paris, 1972, p. $168-169$ et 180 . 
(Villa Dei), telles Vildé-Guingalan, à l'ouest de Dinan, et Vildé-la-Marine, sur la baie du Mont-Saint-Michel ${ }^{77}$.

Implantés dans la plus grande partie du duché de Bretagne, jusqu'en des endroits reculés, les Templiers n'ont jamais recherché les "déserts". Dans les campagnes, ils ont souvent privilégié la proximité des voies de circulation, parfois d'origine romaine, les abords du littoral ou les points de franchissement des rivières, Montbran, en Pléboulle, dans le diocèse de Saint-Brieuc, se situant par exemple là où l'antique route de Carhaix à Alet passait le Frémur ${ }^{78}$. Dans un esprit comparable, bien plus qu'on ne l'estime souvent, ils se sont intéressés aux villes, aux grandes, à Nantes en tout premier lieu, comme à de plus petites, à l'image de La Guerche et de Clisson ${ }^{79}$, où ils furent à l'origine du faubourg de La Madeleine, désigné dans le rentier de 1257 sous le nom de burgum Templi Clicii ${ }^{80}$. Cette implantation urbaine des Templiers ne doit pas surprendre. Outre le fait que les plus grandes étaient des centres d'influence, les villes constituaient des nœuds d'échanges et de communication dont les frères avaient besoin. Elles fournissaient communément des possibilités de stockage et de commercialisation, bien connues dans le cas de Nantes, où le Temple, à la différence de l'Hôpital, était admis depuis 1226 à écouler son vin pendant le ban de l'évêque ${ }^{81}$; parfois, elles pouvaient être le lieu d'activités de prêt ou de dépôt, dont il faut se garder de faire trop vite de la banque ${ }^{82}$. Jouant un rôle primordial de trait d'union, les établissements templiers urbains permettaient à la Bretagne de s'accorder aux missions de l'ordre et d'en intégrer l'organigramme.

\section{L'organisation locale de l'ordre}

Au Temple, le secours de la Terre sainte, qui constituait la raison d'être même de l'ordre, a très tôt supposé une complémentarité étroite entre le front et l'arrière. Ainsi s'est mis en place, à partir de la seconde moitié $\mathrm{du} \mathrm{XII}{ }^{\mathrm{e}}$ siècle, une structure à trois degrés reliant le couvent central, en Orient, à chacune des commanderies occidentales, par le biais d'une circonscription intermédiaire, la province ${ }^{83}$. La Bretagne, où, comme en d'autres régions, était intervenu dans un premier temps le maître cismarin, représentant direct du supérieur de l'ordre, s'intégra à ce schéma autour de 1225, lorsque le maître provincial d'Aquitaine y étendit son autorité, le

77. Colin, François, " Templiers et Hospitaliers en Bretagne », p. 81-84.

78. Gulllotin DE CoRson, Amédée, Les Templiers en Bretagne et les Hospitaliers de SaintJean de Jérusalem, p. 193.

79. Colin, François, "Templiers et Hospitaliers dans les villes de l'ouest de la France (XII ${ }^{\mathrm{e}}$ $\mathrm{XV}^{\mathrm{e}}$ siècle) ", Mémoire de deuxième année de master, Université de Nantes, 2008, t. I, p. 57.

80. LE SAUCE, Xavier, "La commanderie de Clisson au Moyen Âge ", t. I, p. 37.

81. Arch. dép. de la Vienne, 3H 764 (dans un vidimus de l'official d'Angers du 11 juin 1413).

82. Demurger, Alain, Les Templiers. Une chevalerie chrétienne au Moyen Âge, p. 116-129.

83. Ibidem, p. 147-151. 
premier à l'avoir fait, à la faveur d'un accord avec le seigneur de Retz, étant Giraud des Bruyères ${ }^{84}$. Ce dignitaire, dont le ressort, du Bordelais jusqu'au Maine, s'étendait à tout l'Ouest de la France, pouvait le cas échéant s'adjoindre un lieutenant, et c'est sans doute ainsi qu'il faut entendre le rôle de Pierre de Langan, cité en 1245 comme " commandeur des maisons de la chevalerie du Temple en Bretagne ${ }^{85}$ ". Dotées d'une entité véritable au regard de l'ordre, bien qu'elle ne fût jamais fermée - Clisson, par exemple, ayant des liens forts avec le Poitou et l'Anjou -, les terres templières du duché ont pu constituer une baillie, mais elles dépendaient du maître d'Aquitaine, l'unique supérieur de celui-ci étant le grand-maître, présent localement à une reprise au moins, en juin $1293^{86}$.

La visite de Jacques de Molay à Nantes, un an après son élection à Chypre $^{87}$, ressortissait à l'exceptionnel, et en Bretagne, sous l'autorité du maître d'Aquitaine, l'organisation templière était fondée, comme ailleurs, sur un réseau de commanderies qui irriguait le duché. Tout établissement, même puissant, ne formait pas, loin s'en faut, une commanderie, la structure consistant d'abord en un mécanisme institutionnel dont la gestion était déléguée à un frère, le commandeur, qui, pour le bénéfice de l'ordre, se chargeait d'en développer les ressources ${ }^{88}$. Ces centres, dans les textes latins, sont appelés domus ou preceptoria, et, dans les dernières années d'existence du Temple, il semble qu'il en eût en Bretagne entre une demidouzaine et une dizaine (fig. 4$)^{89}$. Nantes formait une commanderie depuis 1202 au moins ${ }^{90}$, son ressort s'étendant à toutes les terres du diocèse au nord de la Loire; au sud, le Vignoble relevait de la maison de Clisson, attestée en $1213^{91}$, et le Pays de Retz de celle des Biais, qu'une matrice de sceau templier du Musée Dobrée, découverte sur place (fig. 5), autorise à penser, passé le milieu du XIII ${ }^{\mathrm{e}}$ siècle $^{92}$, s'être détachée de Coudrie ${ }^{93}$. Hors du diocèse de Nantes, l'unique commanderie absolument avérée est

84. Blanchard, René, Cartulaire des sires de Rays, Poitiers, t. II, 1899, p. 221-222, doc. 203. 85. Arch. dép. de la Vienne, 3H1 784.

86. Barthélemy, Anatole de, et Gesuin de Bourgogne, Jules, Anciens évêchés de Bretagne, t. VI, p. 206-207.

87. LutTrell, Anthony, "The Election of the Templar Master Jacques de Molay ", dans The Debate on the Trial of the Templars (1307-1314), éd. par Jochen Burgtorf, Paul Crawford et Helen Nicholson, Farnham, 2010, p. 21-31.

88. JosserAnd, Philippe, "Commanderie", dans Prier et combattre. Dictionnaire européen des ordres militaires au Moyen Âge, éd. par Nicole Bériou et Philippe Josserand, Paris, 2009, p. 245-246.

89. Colin, François, « Templiers et Hospitaliers en Bretagne », p. 97-100.

90. Barthélemy, Anatole de, et Geslin de Bourgogne, Jules, Anciens évêchés de Bretagne, t. VI, p. 148.

91. LE SAUCE, Xavier, "La commanderie de Clisson au Moyen Âge ", p. 17-18.

92. Rien ne permet d'étayer la datation de DuRU, André, "Templiers, Hospitaliers et Vraie Croix de Saint-Père en Retz ", Bulletin de la Société des Historiens du Pays de Retz, 4 (1984), p. 62, faisant remonter l'établissement templier à la fin du XII ${ }^{\mathrm{e}}$ siècle.

93. Lisle du Dreneuc, Pitre de, Catalogue du Musée Archéologique de Nantes, Nantes, 1903, p. 163. 

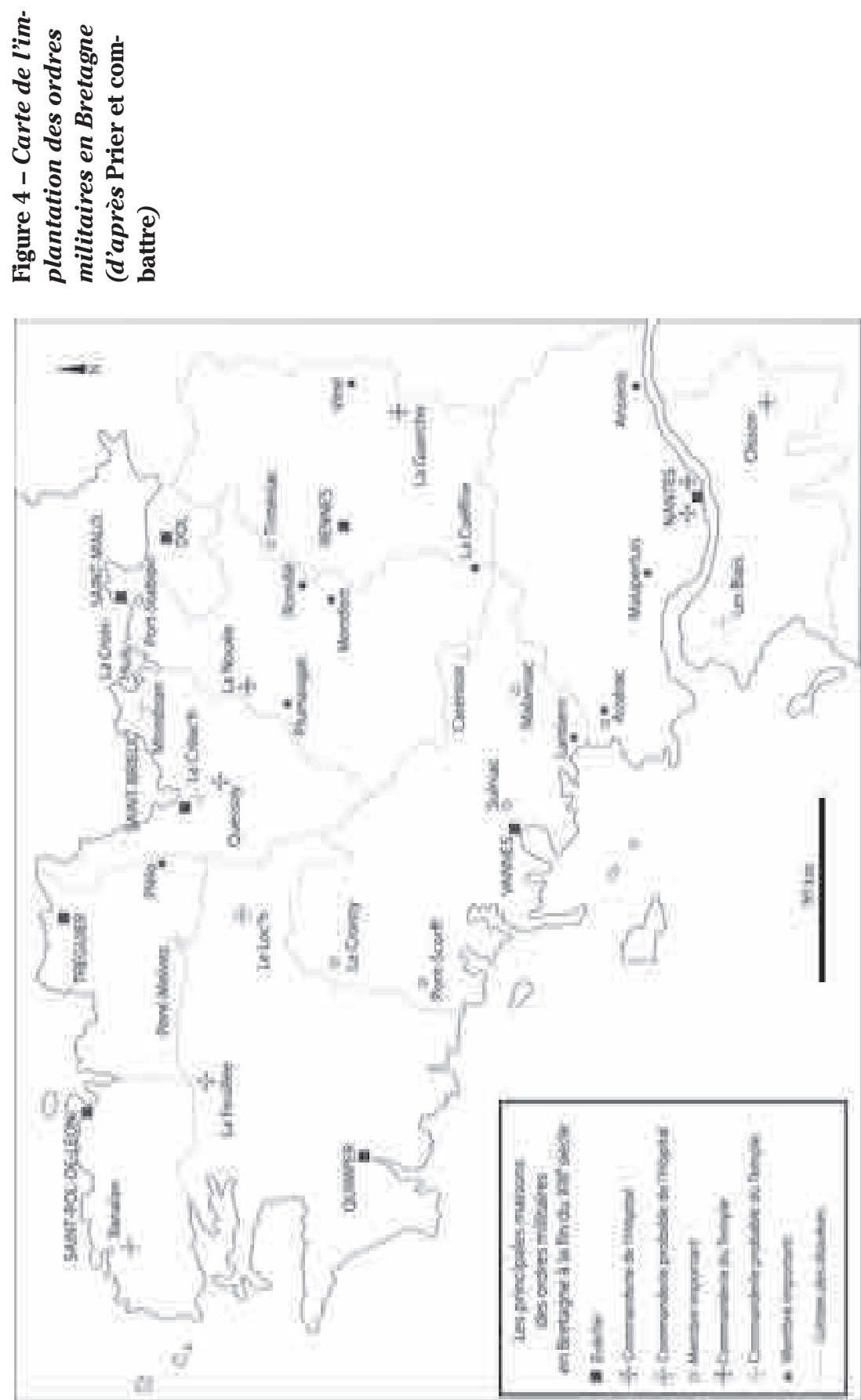
Figure 5 - Dessin du sceau de frère Robert retrouvé aux Biais (d'après F. Parenteau)

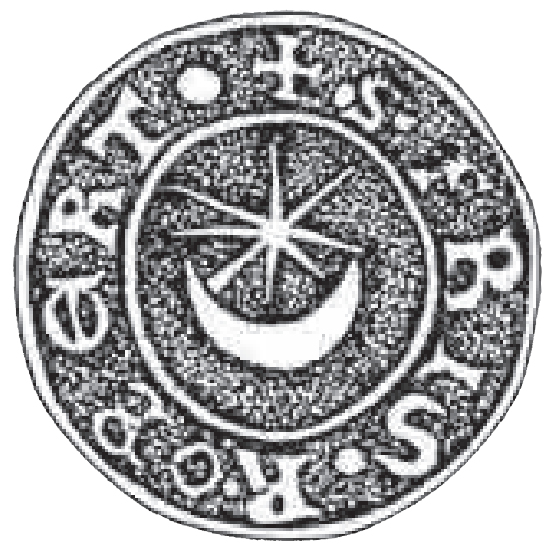

celle de La Guerche, citée en $1245^{94}$, mais il est bien certain que le réseau templier se complétait vers le nord et vers l'ouest avec des maisons, plus que probables, à la Nouée, la Caillibotière et au Créac'h, et d'autres, certes moins assurées, mais possibles, à Carentoir, Montbran et PontMelvez ${ }^{95}$.

Au total, les commanderies bretonnes du Temple n'ont pas dû abriter plus d'une trentaine de frères, la plupart de ceux qui y vivaient étant, comme très souvent en Occident, des familiers et des dépendants. À l'échelle locale, les trois groupes entre lesquels se distribuaient les membres de l'ordre sont attestés, chevaliers, chapelains et sergents. Il se pourrait même qu'ils aient été figurés ensemble sous la forme de neuf personnages agenouillés au départ du fût du grand calvaire édifié, d'après Marc Déceneux, à la toute fin du XIII ${ }^{\mathrm{e}}$ siècle pour l'établissement templier de Saint-Jouan et déplacé en 1774 dans le placître de Saint-Maudez, à l'ouest de Dinan (fig. 6 et 6 bis) ${ }^{96}$. Chevaliers et chapelains, rarement individualisés dans les sources, étaient en Bretagne moins nombreux que les sergents, et ces derniers ont pu assumer la gestion de puissantes commanderies. Issus de la chevalerie, comme Hamelin du Pinel et Pierre de Langan, mais aussi de milieux plus modestes, les Templiers bretons ont servi dans le duché avec des coreligionnaires venus d'autres régions, Picards, Tourangeaux ou Limousins apparaissant par exemple à la faveur des interrogatoires du procès ${ }^{97}$. Hors de Bretagne, à l'inverse, quelques frères qui avaient servi dans le duché ont rejoint l'Orient, non par l'Atlantique - Jean-Christophe Cassard l'a justement noté $^{98}$ - mais par l'axe ligérien, le Rhône et la Méditerranée, achevant au loin de belles carrières, tels Hugues Salomon de Quily et Guillaume II de La Guerche, respectivement maréchal et sénéchal de l'ordre dans le troisième quart du XII ${ }^{\mathrm{e}}$ siècle $^{99}$.

94. Barthélemy, Anatole de, et Geslin de Bourgogne, Jules, Anciens évêchés de Bretagne, t. VI, p. 172.

95. Colin, François, "Templiers et Hospitaliers en Bretagne », p. 99-100.

96. DÉCENEUX, Marc, "Le grand calvaire breton. Une formule née dans le haut pays ", $\mathrm{Ar}$ Men, 132 (2003), p. 34-35.

97. MiCHELET, Jules, Le procès des Templiers, t. II, p. 82 et 105.

98. CASSARD, Jean-Christophe, L'Orient des Bretons au Moyen Âge, p. 147.

99. Burgtorf, Jochen, The Central Convent of Hospitallers and Templars. History, Organization, and Personnel (1099/1120-1310), Leyde, 2008, p. $47-48$ et 439, et MEURET, Jean- 


\section{Traces et mémoires templières}

\section{Le labyrinthe des traditions locales}

L'histoire du Temple en Bretagne est riche, et l'implantation de l'ordre, partout sensible, sauf dans le Léon, a pu localement revêtir une grande importance. On pourrait s'attendre à ce que ces éléments, après la disparition des frères, aient suscité une mémoire abondante, entraînant toute une série de discours et d'écrits autour du passé templier bre-

\section{Figures 6 et 6 bis - Le calvaire des Templiers de Saint-Maudez (cliché Chr. Renault)}
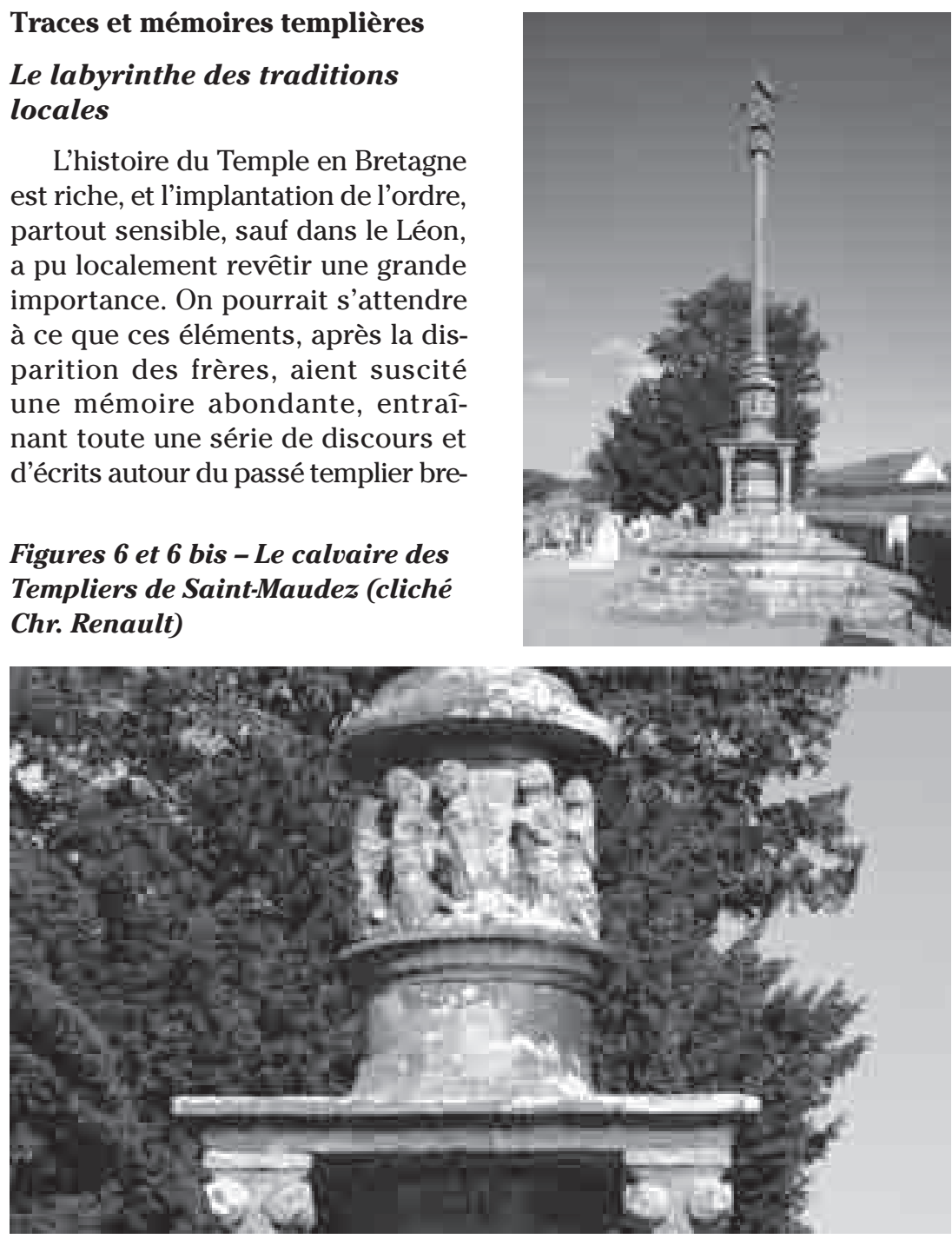

Claude, " Au cœur de la marche bretonne, l'ascension et le démantèlement d'une grande châtellenie, La Guerche-Pouancé (milieu XI ${ }^{\mathrm{e}}$-milieu XII ${ }^{\mathrm{e}}$ siècle) ", dans : Le pouvoir et la foi au Moyen Âge en Bretagne et dans l'Europe de l'Ouest. Mélanges en mémoire du professeur Hubert Guillotel, éd. par Joëlle Quaghebeur et Sylvain Soleil, Rennes, 2010, p. 645-647. Il est à souligner cependant que le premier des deux dignitaires, connu seulement à travers sa pierre tombale, dépourvue de date (DE SANDOLI, Sabino, Corpus inscriptionum crucesignatorum Terrae Sanctae (1099-1291), Jérusalem, 1974, p. 256-257, doc. 346), est appelé Hugues Salomon du Quiliou par CLAVERIE, Pierre-Vincent, L'ordre du Temple en Terre sainte et à Chypre au XIIt siècle, Nicosie, t. II, 2005, p. 78-79, et situé surtout un siècle plus tard dans la chronologie, à partir de l'affirmation de CLERMONT-GANNEAU, Charles, Matériaux inédits pour servir à l'histoire des croisades. Fasc. 5 : nouveaux monuments des croisés recueillis en Terre sainte, Paris, 1884, p. 8. 
ton. C'est ici l'univers des traditions locales liées au Temple qui s'ouvre, un monde sans limites, sur lequel, pour la seule Bretagne, des milliers de pages ont été produites et le sont encore. Prétendre l'épuiser ou en fournir une synthèse serait illusoire, et c'est en historien - chose presque singulière - que je l'aborderai. Du Temple, au soir du Moyen Âge en Bretagne, il semble que l'on ait peu parlé. Dernier chroniqueur de l'époque ducale, Alain Bouchart, dans les Grandes chroniques de Bretagne, publiées en 1514, n'a rapporté que la chute de l'ordre et en a dit, en peu de mots, ce que chacun savait ${ }^{100}$. L'unique trace antérieure d'une mémoire bretonne du Temple date de 1407. Elle provient d'une mention portée cette année-là au dos de l'acte relatant la venue à Nantes, le 10 août 1308, des commissaires de Philippe IV en charge du séquestre des biens de l'ordre. D'après l'ajout, ces officiers auraient été chassés par les bourgeois, au nom des droits du duc ${ }^{101}$. L'épisode est douteux, et sa mémoire, à lire dans le contexte politique tendu du début du $\mathrm{XV}^{\mathrm{e}}$ siècle face au royaume de France, dit très peu du souvenir des Templiers ${ }^{102}$.

Pour l'immense majorité d'entre elles, les traditions qui continuent à circuler en Bretagne à propos du Temple ne sont pas médiévales. Savoir quand elles sont nées est très difficile. Beaucoup ont sans doute été forgées au XIX ${ }^{\mathrm{e}}$ siècle, au moment où elles ont commencé à être consignées par écrit : avec sa fascination de l'histoire, du Moyen Âge en particulier, et son attrait pour le mystère, celui de la foi et bien d'autres, l'époque était favorable ${ }^{103}$, et de nombreux gens d'esprit se sont alors enthousiasmés pour les Templiers ${ }^{104}$. Certaines traditions, toutefois, sont plus anciennes, dont, dès la période moderne, on peut identifier la trace. Dubuisson-Aubenay, qui accomplit en 1636 un grand périple en Bretagne, en a relaté deux dans son itinéraire. À Dinan, l'église des Dominicains aurait, à ce que l'on lui dit, appartenu aux Templiers, " de l'ordre desquels le vulgaire estime avoir été ces trois chevaliers qui étaient de la maison d'Angoulevent, très bonne

100. BouchaRt, Alain, Grandes chroniques de Bretaigne, éd. de Marie-Louise Auger et Gustave Jeanneau, Paris, t. II, 1986, p. 25.

101. MoRICE, Hyacinthe, Mémoires pour servir de preuves, t. I, col. 1217.

102. À ce sujet, il me semble nécessaire de faire preuve de plus de réserve que Cous, François, "Templiers et Hospitaliers en Bretagne ", p. 38, et GAUTIER, Louis Christian, Histoire des Templiers en Bretagne, p. 130-131 et 172, trop prompts à suivre l'opinion traditionnelle, incarnée entre autres par MACÉ DE VAUDORÉ, Jean-François de, Dictionnaire historique, géographique et topographique de Nantes et de l'ancien comté nantais, Nantes, 1836, p. 145.

103. GuIOMAR, Jean-Yves, Le Bretonisme. Les historiens bretons au XIX ${ }^{e}$ siècle, Rennes, 1987, p. 79-82.

104. La PoIX DE Fréminville, Christophe-Paulin de, " Notice sur quelques monuments de l'ordre des Templiers dans le département des Côtes-du-Nord ", Mémoires de la Société Nationale des Antiquaires, 15 (1840), p. 343-344 : "Tout ce qui est relatif à l'histoire de l'ordre du Temple, de cet ordre si justement célèbre par sa gloire et ses malheurs, doit fortement intéresser les personnes qui se livrent à des études historiques relatives à notre patrie. Aussi, dans mes actives investigations de l'ancienne province de Bretagne, je me suis attaché d'une manière toute spéciale à recueillir tous les souvenirs, à examiner tous les monuments qui pouvaient concerner plus ou moins directement l'histoire des chevaliers du Temple." 
jadis et à présent faillie ", dont il a décrit les tombes ${ }^{105}$. À Vannes, c'est l'église des Franciscains, située hors de la ville, dans le fief de Ker, que l'on attribuait aux Templiers, même si l'auteur ne cache pas les doutes que lui inspire cette idée : " D'aucuns veulent dire que c'était un temple et chapelle des Templiers, mais il n'y en a point de preuve ${ }^{106}$. " Dès le XVII ${ }^{\mathrm{e}}$ siècle, des traditions liées au Temple ont donc existé en Bretagne, le cas échéant contestées, et elles ne semblent pas avoir été particulièrement négatives.

Souvent, dans les traditions rapportées depuis le XIX ${ }^{\mathrm{e}}$ siècle, les Templiers sont assimilés à des figures maléfiques. Ce sont ces fameux "moines rouges", sur lesquels on a tellement écrit en Bretagne ${ }^{107}$. Aucun récit n'égale la ballade que Théodore Hersart de La Villemarqué entendit dans la paroisse de Nizon de la bouche d'une mendiante ${ }^{108}$ : contant la fin tragique de Katellig Moal, abusée par trois frères et miraculeusement vengée par l'enfant né de son viol, le gwerz des "Trois moines rouges " (An tri manac'h ruz) renverrait à un épisode situé, à la charnière des XIII et XIV ${ }^{\mathrm{e}}$ siècles, sous l'épiscopat quimpérois d'Alain Morel ${ }^{109}$. De tels récits ne sont pas propres à la Basse-Bretagne, et de semblables ont pu circuler en pays gallo, tel celui qu'a publié Louis-Christian Gautier, sur la base d'une collecte opérée à La Guerche par Michel Lascaux en 1974, mettant en scène, lui aussi, des Templiers criminels, idolâtres et voués à l'enfer ${ }^{110}$. Contrairement à ce que l'on croit d'ordinaire, toutes les légendes sur le Temple en Bretagne ne sont pas négatives, ainsi qu'Amédée Guillotin de Corson l'avait déjà signalé, se faisant l'écho d'un récit favorable, attaché à Montbran, où le grand-maître en personne intervenait au secours d'un voleur repentant qui lui avait sauvé la vie ${ }^{111}$. Terrible ou au contraire propice, la tradition prête d'abord aux Templiers une puissance et en fait de grands bâtisseurs, chapelles, églises, logis, voire châteaux leur étant souvent attribués.

\section{La modestie du bâti conservé}

Le bâti laissé par les Templiers en Bretagne a fait couler infiniment d'encre. Qui sait que Michèle Boccard-Billon, il y a douze ans, a interrompu un projet de recherche sur l'architecture de l'Hôpital dans le duché, faute de témoins archivistiques et monumentaux suffisants ${ }^{112}$, devrait s'en étonner, le Temple étant

105. La Bretagne d'après l'Itinéraire de monsieur Dubuisson-Aubenay, éd. par Alain Croix et al., Rennes, PUR, 2006, p. 225.

106. Ibidem, p. 437.

107. LaSCAuX, Michel, Les Templiers en Bretagne, p. 23-28.

108. Ainsi l'avait déjà souligné GuILLOTIN DE CORSON, Amédée, Les Templiers en Bretagne et les Hospitaliers de Saint-Jean de Jérusalem, p. 324.

109. Hersart de La Villemarqué, Théodore, Barzaz Breiz. Chants populaires de la Bretagne, p. 305.

110. GAUTIER, Louis-Christian, Histoire des Templiers en Bretagne, p. 177-180.

111. Guillotin DE CoRSON, Amédée, Les Templiers en Bretagne et les Hospitaliers de SaintJean de Jérusalem, p. 327-329.

112. BoCCARD-BILlON, Michèle, "L'architecture religieuse des Hospitaliers en Bretagne au Moyen Âge : une étude à entreprendre? ", Bulletin de la Société archéologique du Finistère, 
aussi pauvrement doté que son homologue. Hélas, ceux qui exploitent la veine templière, dans le sillage très fréquenté de Laurent Dailliez ${ }^{113}$, ne s'arrêtent pas à cela, et, en matière architecturale aussi, les traditions locales ont la vie dure : sans trop exagérer, l'on peut dire qu'en dehors des cathédrales, toutes les églises bretonnes susceptibles d'avoir été construites aux XII et XIII ${ }^{\mathrm{e}}$ siècles - et parfois quelques autres - ont été attribuées aux frères. Prétendre lister les sanctuaires qui faussement ou, au mieux, sans preuve ont été tenus pour templiers n'aurait pas de sens. Aussi, avant d'analyser les vestiges assurés de l'ordre en Bretagne, ne ferai-je que dire un mot des causes de tant d'identifications erronées. Outre la confusion entre le Temple et l'Hôpital, qui a joué par exemple au Guerno, les principales tiennent au désir d'ajouter au prestige d'un édifice en le rattachant à l'ordre - Brélevenez, près de Lannion, Lanleff, Merlevenez, Loctudy et tant d'autres - ou, à l'inverse, à l'espoir, critiqué en son temps par Amédée Guillotin de Corson, d'attirer l'intérêt sur un monument peu connu, en en faisant la propriété des frères ${ }^{114}$.

Face à une telle littérature, la réalité historique court le risque de décevoir. Les églises du Temple qui subsistent aujourd'hui en Bretagne ont presque toutes été reconstruites à l'initiative de l'Hôpital, entre le xve et le $\mathrm{XVII}^{\mathrm{e}}$ siècle, dans le style flamboyant développé à partir de la fin de la guerre de Succession $^{115}$. Plusieurs, comme à Carentoir ou au Créac'h, pourraient abriter quelques vestiges antérieurs au XIV ${ }^{\mathrm{e}}$ siècle ${ }^{116}$, mais deux seulement n'ont subi que des modifications minimes et se présentent dans un état proche de celui qui était le leur à l'époque des Templiers. Il s'agit des chapelles de Clisson et de la Nouée. L'une et l'autre sont des constructions romanes très simples. La Madeleine, à Clisson, remarquablement décrite au début du Xxe siècle par Paul de Berthou ${ }^{117}$, est un bel édifice, austère, composé d'une nef de trois travées voûtées en berceau brisé et d'un chevet en hémicycle dont le cul-defour est précédé d'une travée droite; des contreforts scandent l'extérieur, y compris sur la façade, surmontée d'un clocher-mur à deux baies en plein cintre couronné par un fronton triangulaire (fig. 7) ${ }^{118}$. Saint-Jean Baptiste, à la Nouée, présente une nef de plan rectangulaire ouvrant sur une abside en hémicycle éclairée par une fenêtre étroite dont le linteau, solution assez rare d'après Louise-Marie Tillet, est taillé en plein cintre dans un seul bloc

129 (2000), p. 87-96.

113. DallLIEZ, Laurent, La France des Templiers, Paris, 1974.

114. Gullotin de Corson, Amédée, Les Templiers en Bretagne et les Hospitaliers de SaintJean de Jérusalem, p. 302-303, s'est ainsi notablement distancié pour la chapelle de Penesclus de la position de CAYOT-DÉLANDRE, François-Marie, Le Morbihan, son histoire et ses monuments, Vannes, 1847, p. 226, qui écrivait : "On entre à Muzillac par le faubourg de Penesclus, dont la chapelle délabrée n'attirerait pas les regards du voyageur, s'il ne savait qu'elle passe pour avoir appartenu aux Templiers. "

115. BOCCARD-BILLON, Michèle, « L'architecture religieuse des Hospitaliers en Bretagne au Moyen Âge ", p. 89.

116. GAUTIER, Louis-Christian, Histoire des Templiers en Bretagne, p. 90 et 110.

117. BerTHOu, Paul de, Clisson et ses monuments. Étude historique et archéologique, Nantes, 1910, p. 107-119.

118. Tillet, Louise-Marie, Bretagne romane, La-Pierre-Qui-Vire, 1982, p. 52-53. 
(fig. 8) ${ }^{119}$. À Clisson comme à la Nouée, de restauration plus récente ${ }^{120}$, les chapelles reflètent ce " style simple et fonctionnel " dont Alain Demurger a rappelé qu'il était la pratique du Temple ${ }^{121}$.

Figure 7 - Vue générale de la chapelle templière de Clisson

(cliché Chr. Renault)

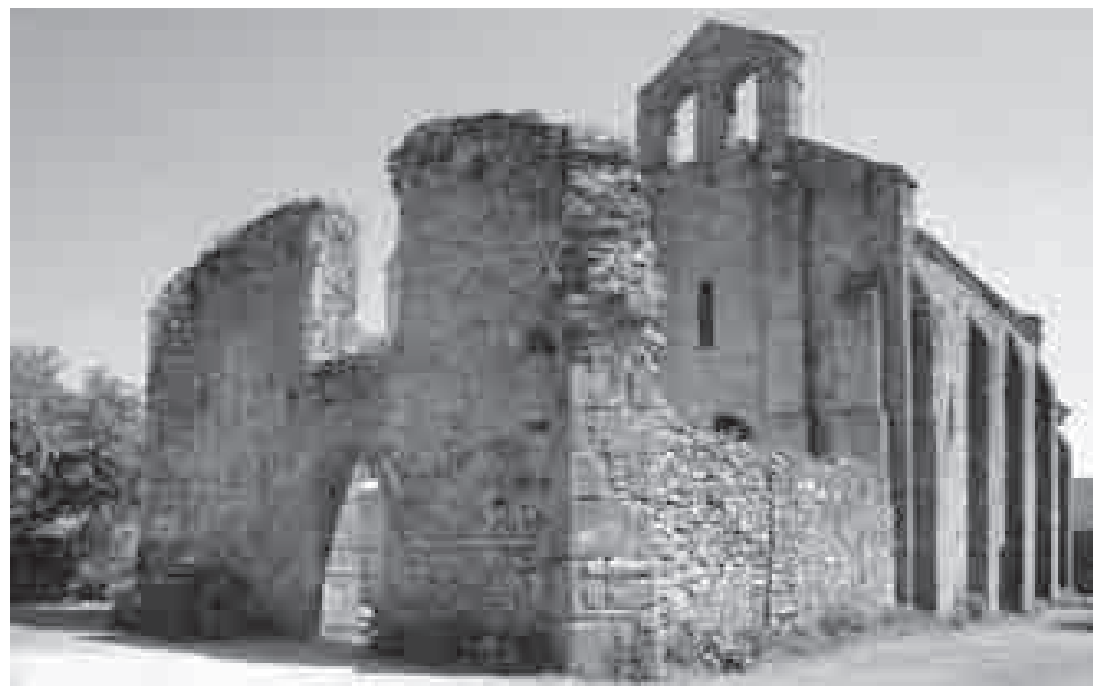

Figure 8 - Vue générale de la chapelle de mla Nouée (cliché Chr. Renault)

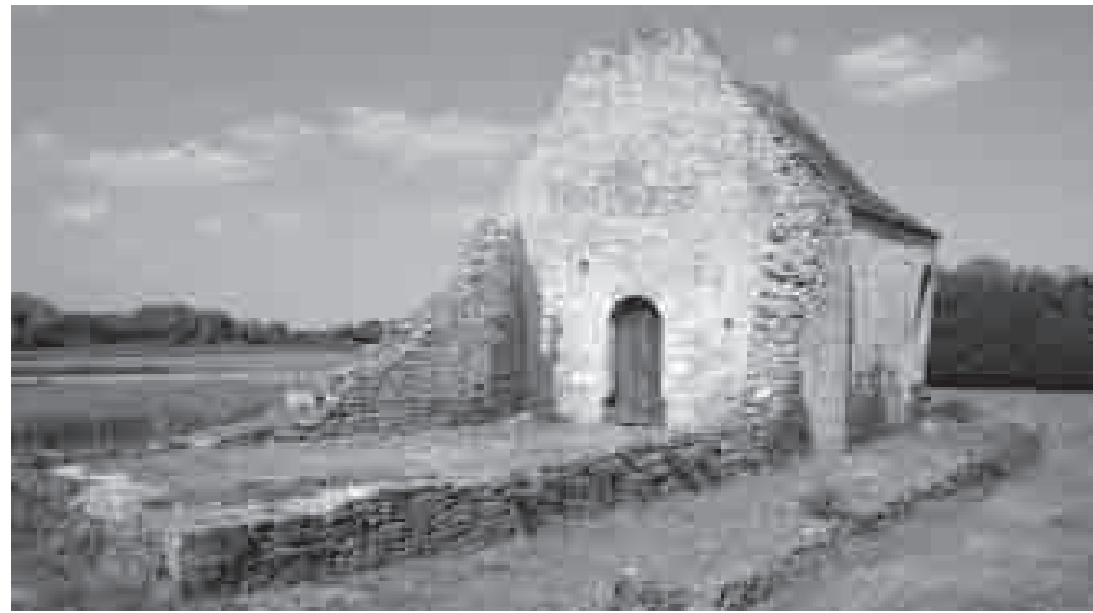

119. Ibidem, p. 64.

120. GAUTIER, Louis Christian, Histoire des Templiers en Bretagne, p. 107-108.

121. Demurger, Alain, Les Templiers. Une chevalerie chrétienne au Moyen Âge, p. 172. 
Si le bâti religieux hérité des Templiers en Bretagne apparaît modeste, les traces de leurs édifices militaires et civils sont encore bien plus maigres. La tradition attribue aux frères plusieurs châteaux, comme le Mur, en SaintÉvarzec $^{122}$, mais cela relève de la légende, y compris à Montbran, où un établissement de l'ordre, qui fut peut-être une commanderie, est avéré. Certes, il existe, au-dessus du Frémur, une tour octogonale, actuellement en ruine, mais rien ne prouve qu'elle ait un lien quelconque avec les frères et qu'elle constitue, comme le laissait à entendre jadis Amédée Guillotin de Corson, le seul exemple d'édifice militaire que l'on puisse attribuer avec certitude aux Templiers en Bretagne ${ }^{123}$. Les commanderies du duché, dénuées de fonctions militaires, étaient, on l'a dit, des centres d'exploitation, où, le plus souvent, un logis était associé à des bâtiments agricoles ${ }^{124}$. De tels édifices, l'on ne conserve pratiquement plus de traces. Certains furent ruinés assez tôt, comme à la Caillibotière, dont, en 1681, il ne subsistait que de "vieilles murailles ", et ils ont depuis totalement disparu ${ }^{125}$. D'autres, comme à La Guerche, ont été profondément remaniés à l'initiative de l'Hôpital, essentiellement sous l'Ancien Régime, et il est vain de vouloir y trouver une mémoire du Temple ${ }^{126}$, à l'instar de Louis-Christian Gautier, enclin à dater du XII ${ }^{\mathrm{e}}$ siècle la porte d'entrée du manoir de la Nouée d'après l'avis de l'actuel propriétaire des lieux ${ }^{127}$.

\section{De l'utilité d'une histoire fondée sur l'écrit}

Trop souvent, ce que l'on peut lire sur les Templiers en Bretagne, sur support imprimé ou électronique, procède de légendes et, peut-on dire, de racontars. Les traditions locales peuvent être utiles à qui s'intéresse au passé du Temple, à condition d'être mises en perspective et analysées de façon critique et équilibrée, mais l'histoire de l'ordre suppose d'être construite à partir de faits, et, à cet égard, l'écrit est indispensable. De Bretagne, d'ailleurs, les Templiers n'ont pas disparu sans laisser d'archives, même si ces dernières sont rares, notamment si l'on les compare avec celles des Mendiants, et conservées aujourd'hui en dehors des limites de l'ancien duché - ce qui n'aide pas à leur consultation par les auteurs pressés. Intégrés après 1312 au fonds du grand-prieuré hospitalier d'Aquitaine, les documents bretons du Temple ont été versés aux Archives départementales de la Vienne à la Révolution française ${ }^{128}$. À Poitiers, ils restent aujourd'hui catalogués comme ils l'étaient au XVIII ${ }^{\mathrm{e}}$ siècle, les actes se

122. GAUTIER, Louis-Christian, Histoire des Templiers en Bretagne, p. 120.

123. Guillotin DE CoRson, Amédée, Les Templiers en Bretagne et les Hospitaliers de SaintJean de Jérusalem, p. 192-193.

124. Demurger, Alain, Les Templiers. Une chevalerie chrétienne au Moyen Âge, p. 163-164. 125. Guillotin DE CoRson, Amédée, Les Templiers en Bretagne et les Hospitaliers de SaintJean de Jérusalem, p. 194.

126. Ibidem, p. 180-181.

127. GAUTIER, Louis-Christian, Histoire des Templiers en Bretagne, p. 108.

128. Gulllotin de CoRson, Amédée, Les Templiers en Bretagne et les Hospitaliers de SaintJean de Jérusalem, p. 23, et CoLın, François, "Templiers et Hospitaliers en Bretagne ", p. 8. 
répartissant entre les quatre commanderies que l'Hôpital détenait alors en Bretagne, Carentoir, La Feuillée, La Guerche et Nantes, à l'exception de ceux de Clisson et des Biais, rattachés aux archives des maisons poitevines de Mauléon et Coudrie ${ }^{129}$. C'est sur ce fonds, déjà fréquenté par les Mauristes durant l'Ancien Régime, que toute étude des Templiers en Bretagne doit prendre appui, d'utiles compléments existant à Paris et dans les dépôts locaux, riches parfois de sources de la fin du Moyen Âge et de l'époque moderne qui éclairent l'histoire plus ancienne.

De l'intérêt de cette recherche en archives et en bibliothèques propre à mettre au jour des documents, y compris iconographiques, renseignant le passé templier breton, on a un bel exemple avec la commanderie de Nantes. Attestée pour la première fois en $1202^{130}$, elle a été jusqu'à la chute de l'ordre la plus importante du duché. Construite sur un terrain, au Pré d'Anian, donné aux frères en 1141, dans le Bourg-Main appelé faubourg Saint-Nicolas ${ }^{131}$, elle fut intégrée à la cité quand Pierre de Dreux, en 1222, développa l'enceinte urbaine sur la rive droite de l'Erdre ${ }^{132}$. Les Templiers reçurent, pour prix de l'empiètement qu'ils acceptèrent, le droit d'appuyer leurs édifices au rempart, et l'on apprend, à cette occasion, que la commanderie, à l'intérieur d'un enclos, comprenait un logis et un cimetière, sans doute déjà associé à une chapelle ${ }^{133}$. Vouée à sainte Catherine, celleci existait selon toute vraisemblance en $1277^{134}$, lorsqu'un acte fit mention de la construction d'un cellier neuf et de travaux de rénovation en cours sur le logis ${ }^{135}$. En 1415, les bâtiments, désormais passés à l'Hôpital, nécessitaient des réparations, et, six ans plus tard, le toit de la chapelle fut refait ${ }^{136}$. Toutefois, à la fin du Moyen Âge, l'ancienne maison du Temple était déchue, et le souvenir même de sa chapelle, que l'on allait exhumer

129. Aux Archives départementales de la Vienne, la documentation provenant des commanderies bretonnes est classée de la sorte : Carentoir (3H1, cartons 300-318 et registres 400-404), La Feuillée (3H1, cartons 464-466 et registres 443-450), La Guerche (3H1 579-621 et registres 494-498) et Nantes (3H1, cartons 764-793 et registres 525-557); à cela s'ajoutent les actes issus des établissements des Biais (3H1, cartons 408-418 et registre 421 bis) et de Clisson (3H1 cartons 729-748 et registres 525-527), dépendant, à la fin de l'Ancien Régime, de Coudrie et de Mauléon. Pour la période templière, on ne dispose que de sources réduites, intéressant La Guerche (3H1 carton 579 et registre 497), Nantes (3H1, cartons 764, 774, 781, 784 et 790 et registre 541) et Clisson (3H1, carton 729).

130. Barthélemy, Anatole de, et Geslin De Bourgogne, Jules, Anciens évêchés de Bretagne, t. VI, p. 148.

131. Morice, Hyacinthe, Mémoires pour servir de preuves, t. I, col. 583.

132. TonNerre, Noël-Yves, " Le haut Moyen Âge ", p. 71-73.

133. MoRICE, Hyacinthe, Mémoires pour servir de preuves, t. I, col. 850-851.

134. Si la chapelle n'apparaît pas dans les sources avant 1450 (Arch. dép. de la Vienne, 3H1 764), sa construction date du XIII ${ }^{\mathrm{e}}$ siècle, d'après OGÉE, Félix-François, " Notice sur l'ancienne chapelle des Templiers ", Le Lycée armoricain, 3.2 (1825), p. 254.

135. Barthélemy, Anatole de, et Geslin de Bourgogne, Jules, Anciens évêchés de Bretagne t. VI, p. 195-196.

136. Arch. dép. de la Vienne, $3 \mathrm{H} 1$ 764, cité par CouIN, François, " Templiers et Hospitaliers dans les villes de l'ouest de la France ", t. I, p. 31. 
en 1825 , lors du percement de l'actuelle rue d'Orléans (fig. 9), se perdit ${ }^{137}$, l'Hôpital en faisant bâtir une nouvelle, elle-même démolie en 1771 avec les bâtiments adjacents, lorsque fut créé l'alignement qui forme aujourd'hui le quai Brancas ${ }^{138}$. De nos jours, à Nantes, en dehors des noms des rues du Pré-Nian et Sainte-Catherine, il ne reste aucune mémoire templière, mais les écrits sont là, qui, en l'absence même de bâti, permettent de la faire revivre ${ }^{139}$.

\section{Figure 9 - Extrait du plan archéologique de Nantes par Henri Soreau (cliché Soc. Archéologique de Nantes et de Loire-Atlantique)}

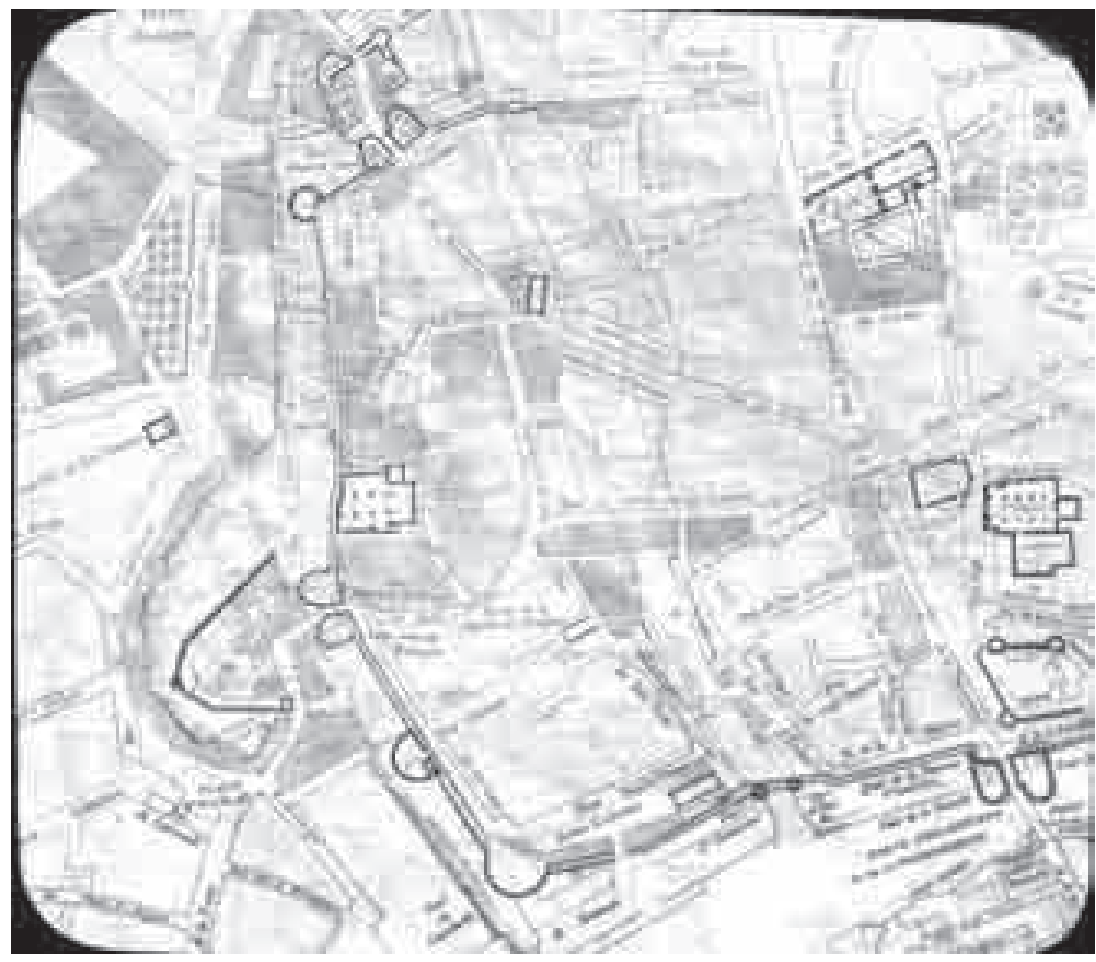

L'histoire des Templiers en Bretagne, dans la première décennie du $\mathrm{XXI}^{\mathrm{e}}$ siècle, a avancé de façon substantielle et, comme le manifeste l'exemple

137. OGÉE, Félix-François, " Notice sur l'ancienne chapelle des Templiers », p. 252-255.

138. PÉROT, Élisabeth, "Évolutions et traces des territoires / édifices religieux à Nantes du milieu du XVIII ${ }^{\mathrm{e}}$ siècle à nos jours ", Mémoire de DEA, Université de Versailles-SaintQuentin-en-Yvelines et École d'architecture de Versailles, 2005, p. 32 et 37.

139. Josserand, Philippe, "Templiers ", dans Dictionnaire de Nantes, éd. par Alain Croix et al., à paraître en 2013. 
de l'implantation de l'ordre à Nantes, elle peut continuer à le faire dans les années à venir. Aux historiens, il incombe la responsabilité, dans ce domaine comme dans d'autres, de divulguer le fruit de leurs recherches auprès du public. Peut-on souhaiter en retour que ce dernier sache distinguer entre les fictions, de tous ordres, et la réalité historique et qu'il accorde sa confiance aux spécialistes plutôt qu'aux charlatans ${ }^{140}$ ? Dès qu'il s'agit du Temple - et en Bretagne, peut-être, plus qu'ailleurs -, les légendes, depuis le $\mathrm{xIX}^{\mathrm{e}}$ siècle, sont promptes à courir. Dans bien des publications, imprimées ou électroniques, centrées sur les frères, l'ésotérisme reste présent. Aux supputations de Nicole Villeroux, écrivant " pour ceux qui peuvent soulever le voile et lire le silence des pierres ${ }^{141}$ ", qui s'interrogeait, voici trente ans, sur la présence du Baphomet, l'idole supposée des frères, au calvaire du Champ de la Croix rouge, en Plomodiern ${ }^{142}$, répondent aujourd'hui les certitudes de bien des internautes. Et voici comment un monument, très postérieur au XIV ${ }^{\mathrm{e}}$ siècle, devient templier... La communication touristique est un autre fléau, et, à travers des supports qui ne cessent de se multiplier - brochures, ronds-points routiers ou portails informatiques - nombre de collectivités contribuent à brouiller et à fausser l'histoire des Templiers. Puissent ces pages constituer un jalon et susciter l'envie de mieux connaître le passé d'un ordre dont l'intérêt, en Bretagne comme ailleurs, devrait définitivement l'emporter sur l'attrait de l'occulte.

140. Je rejoins ici la question posée par DEMURGER, Alain, " Histoire de l'historiographie des ordres religieux-militaires de 1500 à nos jours ", dans Prier et combattre. Dictionnaire européen des ordres militaires au Moyen Âge, éd. par Nicole Bériou et Philippe Josserand, Paris, 2009, p. 45-46.

141. VILLEROUX, Nicole, "Les Templiers en Bretagne ", dans Sur les pas des Templiers en Bretagne, Normandie et Pays de Loire, éd. par Michel Dumontier, Nicole Villeroux, Georges Bernage et Thierry Barreau, Paris, 1980, p. 60.

142. Ibidem, p. 66-67 : "La sacristie de la chapelle de Sainte-Marie du Menez-Hom porte le nom de Chambre des moines rouges (Kambr ar venec'h ru). Plus loin, on trouve le Champ de la croix rouge (Park ar groaz ru). Le calvaire qui s'élève dans l'enclos de SainteMarie est curieusement orienté : le crucifié est face au couchant et le Christ en majesté face au levant. Au Champ de la croix rouge, sur le calvaire, un ange présente la Sainte Face au levant : est-ce le Saint-Suaire? la tête qu'auraient adorée les Templiers? ". 